\title{
Physics
}

Physics Research Publications

\section{Multiwavelength observations of Markarian 421 in 2001 March: An unprecedented view on the $\mathrm{X}$-ray/TeV correlated variability}

G. Fossati, J. H. Buckley, I. H. Bond, S. M. Bradbury, D. A. Carter-Lewis, Y. C. K. Chow, W. Cui, A. D. Falcone, J. P. Finley, J. A. Gaidos, J. Grube, J. Holder, D. Horan, D. Horns, M. M. Jordan, D. B. Kieda, J. Kildea, H. Krawczynski, F. Krennrich, M. J. Lang, S. LeBohec, K. Lee, P. Moriarty, R. A. Ong, D. Petry, J. Quinn, G. H. Sembroski, S. P. Wakely, and T. C. Weekes 
The Astrophysical Journal, 677:906-925, 2008 April 20

(C) 2008. The American Astronomical Society. All rights reserved. Printed in U.S.A.

\title{
MULTIWAVELENGTH OBSERVATIONS OF MARKARIAN 421 IN 2001 MARCH:
} AN UNPRECEDENTED VIEW ON THE X-RAY/TeV CORRELATED VARIABILITY

\author{
G. Fossati, ${ }^{1}$ J. H. Buckley, ${ }^{2}$ I. H. Bond, ${ }^{3}$ S. M. Bradbury, ${ }^{3}$ D. A. Carter-Lewis, ${ }^{4}$ Y. C. K. Chow, ${ }^{5}$ W. Cui, ${ }^{6}$ \\ A. D. Falcone, ${ }^{7}$ J. P. Finley, ${ }^{6}$ J. A. Gaidos,${ }^{6}$ J. Grube, ${ }^{3}$ J. Holder,${ }^{8}$ D. Horan, ${ }^{9,10}$ D. Horns,${ }^{11}$ \\ M. M. Jordan, ${ }^{12}$ D. B. Kieda, ${ }^{13}$ J. Kildea, ${ }^{9}$ H. Krawczynski, ${ }^{2}$ F. Krennrich, ${ }^{4}$ M. J. Lang, ${ }^{14}$ \\ S. LeBohec, ${ }^{13}$ K. Lee, ${ }^{2}$ P. Moriarty, ${ }^{15}$ R. A. Ong, ${ }^{5}$ D. Petry, ${ }^{4,16}$ J. Quinn, ${ }^{17}$ \\ G. H. Sembroski, ${ }^{6}$ S. P. Wakely, ${ }^{18}$ and T. C. Weekes ${ }^{9}$ \\ Received 2007 July 12; accepted 2007 October 18
}

\begin{abstract}
We present a detailed analysis of week-long simultaneous observations of the blazar Mrk 421 at 2-60 keV X-rays (RXTE) and TeV $\gamma$-rays (Whipple and HEGRA) in 2001. Accompanying optical monitoring was performed with the Mt. Hopkins 48 inch telescope. The unprecedented quality of this data set enables us to establish the existence of the correlation between the TeV and X-ray luminosities, and also to start unveiling some of its characteristics, in particular its energy dependence and time variability. The source shows strong variations in both X-ray and $\gamma$-ray bands, which are highly correlated. No evidence of an X-ray $/ \gamma$-ray interband lag $\tau$ is found on the full week data set, with $\tau \lesssim 3 \mathrm{ks}$. A detailed analysis of the March 19 flare, however, reveals that data are not consistent with the peak of the outburst in the $2-4 \mathrm{keV} X$-ray and TeV band being simultaneous. We estimate a $2.1 \pm 0.7 \mathrm{ks} \mathrm{TeV}$ lag. The amplitudes of the X-ray and $\gamma$-ray variations are also highly correlated, and the TeV luminosity increases more than linearly with respect to the $\mathrm{X}$-ray one. The high degree of correlation lends further support to the standard model in which a unique electron population produces the X-rays by synchrotron radiation and the $\gamma$-ray component by inverse Compton scattering. However, the finding that for the individual best observed flares the $\gamma$-ray flux scales approximately quadratically with respect to the X-ray flux poses a serious challenge to emission models for $\mathrm{TeV}$ blazars, as it requires rather special conditions and/or fine tuning of the temporal evolution of the physical parameters of the emission region. We briefly discuss the astrophysical consequences of these new findings in the context of the competing models for the jet emission in blazars.
\end{abstract}

Subject headings: BL Lacertae objects: individual (Mrk 421) — galaxies: active — galaxies: jets — gamma rays: observations — radiation mechanisms: nonthermal X-rays: individual (Mrk 421)

\section{INTRODUCTION}

Mrk 421 is the brightest BL Lac object in the X-ray and UV sky and the first extragalactic source detected at $\mathrm{TeV}$ energies (Punch et al. 1992). Like most blazars, its spectral energy distribution shows two smooth broadband components (e.g., Sambruna et al. 1996; Ulrich et al. 1997; Fossati et al. 1998). The first one extends from radio to X-rays with a peak in the soft to medium X-ray range; the second one extends up to the $\mathrm{GeV}$ to $\mathrm{TeV}$ energies, with a peak presumed to be around $100 \mathrm{GeV}$. The emission up to X-rays is thought to be due to synchrotron radiation from highenergy electrons, while the origin of the luminous $\gamma$-ray radiation is more uncertain. Possibilities include inverse Compton (IC) scattering of synchrotron (synchrotron self-Compton, SSC) or

\footnotetext{
1 Department of Physics and Astronomy, Rice University, Houston, TX 77005; gfossati@rice.edu.

2 Department of Physics, Washington University, St. Louis, MO 63130; buckley@wuphys.wustl.edu.

3 School of Physics and Astronomy, University of Leeds, Leeds, LS2 9JT, $\mathrm{UK}$.

4 Department of Physics and Astronomy, Iowa State University, Ames, IA 50011.

5 Department of Physics and Astronomy, University of California, Los Angeles, CA 90095 .

6 Department of Physics, Purdue University, West Lafayette, IN 47907.

7 Department of Astronomy and Astrophysics, Pennsylvania State University, University Park, PA 16802.

8 Department of Physics and Astronomy, University of Delaware, Newark, DE 19716
}

ambient photons (external Compton, EC) off a single electron population thus accounting for the spectral similarity of the two components (e.g., Macomb et al. 1995; Mastichiadis \& Kirk 1997; Tavecchio et al. 1998; Maraschi et al. 1992; Sikora et al. 1994; Dermer et al. 1992). Alternative hadronic models produce $\gamma$-rays from protons, either directly (proton synchrotron) or indirectly (e.g., synchrotron from a second electron population produced by a cascade induced by the interaction of high-energy protons with ambient photons; Mücke et al. 2003; Böttcher \& Reimer 2004). The synchrotron proton scenario may be more favorable for objects such as Mrk 421 (Mücke et al. 2003) because of the lower density of the diffuse photon fields necessary for processes such as pion photoproduction to be effective. Moreover, it is generally true that hadronic models need a higher level of tuning in order to reproduce the observed highly correlated X-ray $/ \gamma$-ray variability. Hence, in this paper we have not addressed this class of

\footnotetext{
9 Fred Lawrence Whipple Observatory, Harvard-Smithsonian Center for Astrophysics, P.O. Box 97, Amado, AZ 85645.

10 Argonne National Laboratory, Argonne, IL 60439.

11 Institut für Experimentalphysik, Universität Hamburg, Germany.

12 Raytheon Company, Tucson, AZ 85706.

13 Department of Physics, University of Utah, Salt Lake City, UT 84112.

14 Department of Physics, National University of Ireland, Galway, Ireland.

15 School of Science, Galway-Mayo Institute of Technology, Galway, Ireland.

16 Max-Planck-Institut für Extraterrestrische Physik, D-85741 Garching, Germany.

17 School of Physics, University College Dublin, Belfield, Dublin 4, Ireland.

18 Enrico Fermi Institute, University of Chicago, Chicago, IL 60637.
} 
models, and instead focused our limited modeling effort on the pure SSC model.

All of the above models of the $\gamma$-ray emission from blazars have had some degree of success in reproducing both single-epoch spectral energy distributions and their relative epoch-to-epoch changes (Von Montigny et al. 1995, Ghisellini et al. 1998). These favor the SSC model in Mrk 421 because it is a BL Lac object for which the ratio of thermal (accretion disk and broad-line region) and synchrotron photons is $\sim 0.1$, indicating that the EC mechanism is not important. Detailed modeling of blue BL Lac objects finds that the one-component SSC model can generally account for the time-averaged spectral energy distributions (Ghisellini et al. 1998). Some data sets seem to require modifications of the simple model, introducing either multiple SSC components or additional external seed photons (e.g., Błażejowski et al. 2005).

We can, however, further decrease the degeneracy among proposed physical models by taking advantage of blazars' rapid, largescale time variability with simultaneous X-ray/ TeV monitoring (e.g., Tavecchio et al. 1998; Maraschi et al. 1999; Krawczynski et al. 2001). Different models produce emission at a given frequency with particles of different energies, cooling times, and cross sections for different processes (e.g., Blumenthal \& Gould 1970; Coppi \& Blandford 1990), and thus are in principle distinguishable (Krawczynski et al. 2002). For example, the SSC model predicts nearly simultaneous variations in both the synchrotron and Compton components (but see $\S 4$ ), while other models predict more complicated timing (e.g., Ulrich et al. 1997).

With the possible exception of the X-ray and $\gamma$-ray data taken on Mrk 501, the multiwavelength observations on which we base our inferences have often undersampled the intrinsic variability timescales (Buckley et al. 1996; Petry et al. 2000; Tanihata et al. 2001; Maraschi et al. 1999) and lack a sufficiently long baseline to make a quantitative assertion about the statistical significance of a correlation. Recently, there has even been evidence of an orphan TeV flare for the blazar 1ES 1959+650 (Krawczynski et al. 2004), a transient $\gamma$-ray event that was not accompanied by an obvious X-ray flare in simultaneous data.

For what concerns Mrk 421, there have been regular multiwavelength campaigns in the last several years, planned with an observing strategy focusing on month-long timescales (Błażejowski et al. 2005; Rebillot et al. 2006), and in turn a relatively sparse time sampling (typically one Rossi X-Ray Timing Explorer [RXTE] snapshot per night, plus binned $R X T E$ All-Sky Monitor [ASM] light curves). These campaigns showed that X-ray and $\gamma$-ray brightnesses vary in step and that there is certainly a loose correlation, and also raised some questions about the need to consider additional components to account for the spectra (Błażejowski et al. 2005) or very high Doppler factors (Rebillot et al. 2006).

The 2001 March campaign remains the experiment with the highest density coverage at both X-ray and TeV energies and thus the best data set to address questions concerning the characteristics of the variability of the two spectral energy distribution (SED) components. Moreover, the brightness state achieved during the week of the observations was unprecedented and remains unparalleled. Preliminary results were presented in Jordan et al. (2001) and Fossati et al. (2004).

In this paper we present the summary of the multiwavelength observations, with a particular focus on the correlated X-ray/TeV variability, also including the TeV data taken by the HEGRA (High Energy Gamma Ray Astronomy) telescope. An account of the HEGRA 2001 March observations was published by the HEGRA collaboration (Aharonian et al. 2002). Giebels et al. (2007) report on additional TeV observations with the CAT observatory (Cerenkov Array at Themis), simultaneous with the
$R X T E$ data, not included in this paper. The $R X T E$ observations, timing, and spectral properties are fully presented in Fossati et al. (2008, in preparation, hereafter F08).

The paper is organized as follows: The relevant information about the X-ray and TeVobservations and data reduction is given in $\S 2$. The observational findings are presented in $\S 3$ and discussed in $\S 4$ in the context of the synchrotron self-Compton model. Section 4 also summarizes the conclusions.

\section{OBSERVATIONS AND DATA REDUCTION}

\section{1. $R X T E$}

RXTE observed Mrk 421 in the spring of 2001, during Cycle 6 for an approved exposure time of $350 \mathrm{ks}$ (ObsID 60145 [P/60145-01]). Observations started on 2001 March 18 and lasted until 2001 April 1, yielding a total of 48 pointings. The $R X T E$ sampling was very dense until March 25. During the second week, $R X T E$ observed Mrk 421 only during the visibility times for Whipple, whose visibility windows were also drastically shortening. In this paper we only present and discuss the data obtained between March 18 and 25. The journal of this subset of $R X T E$ observations is reported in Table 1. A complete account of the X-ray campaign is presented in F08.

There are two pointed instruments on board $R X T E$, the Proportional Counter Array (PCA; Jahoda et al. 1996) and the HighEnergy X-ray Timing Experiment (HEXTE; Rothschild et al. 1998). The PCA consists of a set of 5 co-aligned xenon/methane (with an upper propane layer) Proportional Counter Units (PCUs) with a total effective area of $\approx 6500 \mathrm{~cm}^{2}$. The instrument is sensitive in the energy range from 2 to $\approx 100 \mathrm{keV}$ (Jahoda et al. 1996). The background spectrum in the PCA is modeled by matching the background conditions of the observation with those in various model files on the basis of the changing orbital and instrumental parameters.

Since the source was very bright throughout the whole campaign, we used bright source selection criteria ${ }^{19}$ to select good time intervals (GTIs) and all layers of the proportional counter units. We did not include the PCU 0 data ${ }^{20}$ in this analysis.

The total exposure time for the PCA was $\sim 263 \mathrm{ks}$, divided over 98 pointing segments, i.e., intervals with the same PCUs on, with individual exposure times ranging between $144 \mathrm{~s}$ and $3.3 \mathrm{ks}$ (we rejected 4 GTIs lasting only $16 \mathrm{~s}$ ). The number of PCUs operational during each pointing varied between 1 and 2 (excluding PCU 0), with the vast majority of cases (97/98) having 2 (for details please refer to F08).

HEXTE consists of two clusters of four $\mathrm{NaI}(\mathrm{T} 1) / \mathrm{CsI}(\mathrm{Na})$ phoswich scintillation counters that are sensitive in the range $15-250 \mathrm{keV}$. Its total effective area is $\approx 1600 \mathrm{~cm}^{2}$. The HEXTE modules are alternately pointed every $32 \mathrm{~s}$ at source and background (offset) positions, to provide a direct measurement of the background during the observation, therefore allowing background subtraction with high sensitivity to time variations in the particle flux at different positions in the spacecraft orbit. Thus, no calculated background model is required. The GTIs for the HEXTE data have been prepared independently, i.e., not requiring any PCU to be active, thus resulting in slightly different on-source times (see Table 1). The total HEXTE on-source time was $\sim 282 \mathrm{ks}$.

The standard data from both instruments were used for the accumulation of spectra and light curves with $16 \mathrm{~s}$ time resolution.

\footnotetext{
19 These criteria are OFFSET $<0.02$, ELV $>10$, and TIME_SINCE_ $\mathrm{SAA}>5$.

20 In 2000 May the propane layer of PCU 0 was lost, resulting in a significantly higher background rate and calibration uncertainty.
} 
TABLE 1

Journal of $R X T E$ Observations

\begin{tabular}{|c|c|c|c|c|c|c|}
\hline \multirow[b]{2}{*}{ Obs. No. } & \multicolumn{2}{|c|}{ Date (UTC) } & \multicolumn{2}{|c|}{ PCA } & \multicolumn{2}{|c|}{ HEXTE } \\
\hline & Start & Stop & $T_{\exp }$ & No. GTIs & $T_{\mathrm{on}}{ }^{\mathrm{a}}$ & No. GTIs \\
\hline 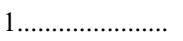 & Mar 18 01:21:40 & Mar 19 01:05:40 & 16544 & 6 & 16544 & 6 \\
\hline 2 & Mar 19 01:05:40 & Mar 19 01:06:11 & 1472 & 1 & 1824 & 1 \\
\hline З & Mar 19 01:06:55 & Mar 19 01:14:49 & 15056 & 5 & 16400 & 5 \\
\hline $4 \ldots \ldots \ldots \ldots \ldots \ldots . .$. & Mar 19 01:14:49 & Mar 19 01:16:17 & 3280 & 1 & 3280 & 1 \\
\hline 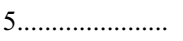 & Mar 19 01:18:13 & Mar 19 01:20:36 & 5488 & 2 & 5984 & 2 \\
\hline 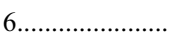 & Mar 19 01:21:19 & Mar 20 01:05:19 & 16464 & 6 & 16464 & 6 \\
\hline 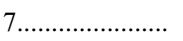 & Mar 20 01:05:19 & Mar 20 01:06:05 & 2144 & 1 & 2640 & 1 \\
\hline 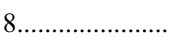 & Mar 20 01:06:59 & Mar 20 01:08:11 & 1936 & 1 & 2576 & 1 \\
\hline 9............................. & Mar 20 01:08:31 & Mar 20 01:13:00 & 8432 & 3 & 9360 & 3 \\
\hline $10 \ldots \ldots \ldots \ldots \ldots \ldots \ldots$ & Mar 20 01:13:29 & Mar 20 01:15:26 & 4560 & 2 & 4560 & 2 \\
\hline $11 \ldots \ldots \ldots \ldots$ & Mar 20 01:16:20 & Mar 20 01:18:51 & 5984 & 2 & 6352 & 2 \\
\hline $12 \ldots \ldots \ldots \ldots \ldots \ldots \ldots$ & Mar 20 01:21:07 & Mar 21 01:05:07 & 16544 & 6 & 16544 & 6 \\
\hline $13 \ldots \ldots \ldots \ldots \ldots \ldots$ & Mar 21 01:05:07 & Mar 21 01:05:58 & 2304 & 1 & 2832 & 1 \\
\hline 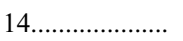 & Mar 21 01:06:40 & Mar 21 01:14:32 & 14720 & 5 & 16448 & 5 \\
\hline $15 \ldots \ldots \ldots \ldots \ldots \ldots \ldots$ & Mar 21 01:14:32 & Mar 21 01:19:01 & 9440 & 3 & 9888 & 3 \\
\hline 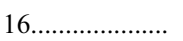 & Mar 21 01:22:52 & Mar 22 01:02:42 & 8784 & 4 & 8784 & 3 \\
\hline $17 \ldots \ldots \ldots \ldots \ldots \ldots$ & Mar 22 01:03:32 & Mar 22 01:04:02 & 1760 & 1 & 1760 & 1 \\
\hline 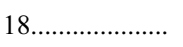 & Mar 22 01:05:19 & Mar 22 01:05:48 & 960 & 1 & 1600 & 1 \\
\hline 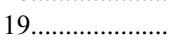 & Mar 22 01:06:41 & Mar 22 01:12:45 & 10656 & 4 & 12560 & 4 \\
\hline 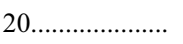 & Mar 22 01:13:13 & Mar 22 01:14:20 & 2112 & 1 & 2112 & 1 \\
\hline $21 \ldots \ldots \ldots \ldots \ldots \ldots$ & Mar 22 01:16:07 & Mar 22 01:16:46 & 2280 & 1 & 2280 & 1 \\
\hline $22 \ldots \ldots \ldots \ldots \ldots \ldots$ & Mar 22 01:19:19 & Mar 22 01:23:48 & 9632 & 3 & 9632 & 3 \\
\hline $23 \ldots \ldots \ldots \ldots \ldots \ldots$ & Mar 23 01:00:07 & Mar 23 01:03:50 & 8456 & 3 & 8456 & 3 \\
\hline 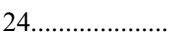 & Mar 23 01:06:24 & Mar 23 01:14:15 & 14368 & 5 & 16368 & 5 \\
\hline $25 \ldots \ldots \ldots \ldots \ldots$ & Mar 23 01:14:15 & Mar 23 01:18:25 & 9512 & 3 & 9784 & 3 \\
\hline 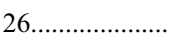 & Mar 23 01:19:06 & Mar 24 01:02:21 & 16296 & 5 & 16296 & 5 \\
\hline $27 \ldots \ldots \ldots \ldots \ldots \ldots$ & Mar 24 01:03:02 & Mar 24 01:03:51 & 2256 & 1 & 2672 & 1 \\
\hline 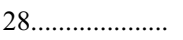 & Mar 24 01:04:41 & Mar 24 01:10:54 & 9984 & 4 & 12784 & 5 \\
\hline $29 \ldots \ldots \ldots \ldots \ldots \ldots$ & Mar 24 01:11:21 & Mar 24 01:14:54 & 7744 & 3 & 7856 & 3 \\
\hline 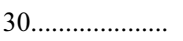 & Mar 24 01:15:45 & Mar 24 01:16:37 & 2880 & 1 & 2880 & 1 \\
\hline $31 \ldots \ldots \ldots \ldots \ldots \ldots$ & Mar 24 01:19:01 & Mar 24 01:21:06 & 5064 & 2 & 5064 & 2 \\
\hline 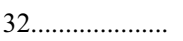 & Mar 24 01:22:26 & Mar 25 01:01:52 & 7448 & 3 & 7448 & 3 \\
\hline 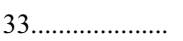 & Mar 25 01:03:12 & Mar 25 01:03:42 & 1152 & 1 & 1680 & 1 \\
\hline $34 \ldots \ldots \ldots \ldots \ldots \ldots \ldots$ & Mar 25 01:04:55 & Mar 25 01:05:49 & 832 & 1 & 1616 & 2 \\
\hline 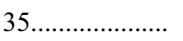 & Mar 25 01:06:27 & Mar 25 01:13:57 & 13024 & 5 & 15216 & 5 \\
\hline 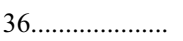 & Mar 25 01:13:57 & Mar 25 01:15:33 & 3296 & 1 & 3296 & 1 \\
\hline Total .............. & Mar 18 01:21:40 & Mar 25 01:15:33 & 262864 & 98 & 281840 & 99 \\
\hline
\end{tabular}

${ }^{\text {a }}$ Not dead-time corrected, and therefore strictly not an exposure time, but an on-source time.

Spectra and light curves were extracted with FTOOLS, version 5.1. PCA background models were generated with the tool pcabackest, from $R X T E$ Guest Observer Facility (GOF) calibration files for bright sources.

The analysis described in the following is mostly based on the 2-15 keV data from the PCA. However, the exceptional brightness of Mrk 421 during this campaign makes it possible to accumulate a light curve with good time resolution also for the higher energy data from the HEXTE detector. HEXTE data between 20 and $60 \mathrm{keV}$ were used.

Light curves for intervals with different active PCUs have been rescaled to the same count per second per PCU units by using the relative weight for the different effective areas as discussed in the $R X T E$ GOF Web site.

The very high count rate in the $R X T E$ PCA enables us to obtain high-signal-to-noise-ratio light curves for several different energy bands, and this to study the energy dependence of the flux variability and $\mathrm{X}-\mathrm{ray} / \mathrm{TeV}$ relationship. Based on the distribution of counts (averaged over the range of observed spectral variability) and the relative background contribution at different energies, we selected the following four bands (expressed in terms of the hardware channels) that have approximately the same statistics: channels 5-8 (labeled 2-4 keV), channels 10-12 (4-6 keV), channels 14-18 (6-8 keV), and channels 20-37 (9-15 keV). Each band comprises on average $\sim 17 \%-24 \%$ of the PCA counts. For more details please refer to F08.

We include here some limited discussion involving HEXTE data (namely, their brightness correlation with the TeV data set).

A nonvariable neutral hydrogen column density $N_{\mathrm{H}}$ of $1.61 \times$ $10^{20} \mathrm{~cm}^{-2}$ (Lockman \& Savage 1995) has been used. However, since the PCA bandpass starts at about $3 \mathrm{keV}$, the adopted value for $N_{\mathrm{H}}$ does not significantly affect our results.

\subsection{Whipple Observatory $\gamma$-Ray Data}

The TeV data on Mrk 421 were taken with the Whipple $10 \mathrm{~m}$ atmospheric Cerenkov telescope. The Whipple telescope detects $\gamma$-rays by imaging the flashes of atmospheric Cerenkov light emitted by $\gamma$-ray-induced electromagnetic showers. Individual shower images are recorded on a photomultiplier tube (PMT) camera and later analyzed to reject the cosmic-ray background, select $\gamma$-ray events, and determine the point of origin and energy of each detected $\gamma$-ray. For the period of the 2001 Mrk 421 observations, 
the camera consisted of a hexagonal array of 490 PMTs on a $0.12^{\circ}$ pitch PMT camera (only the inner 379 pixels were used for the present analysis; Finley et al. 2001). Images were characterized by calculating the moments of the angular distribution of light registered by the PMT camera. These moments include the rms width and length of the shower images and orientation of the roughly elliptical images. Background cosmic-ray showers are rejected using data cuts based on these parameters according to the procedure described by de la Calle Pérez et al. (2003). The point of origin of each detected $\gamma$-ray can be determined from the orientation and elongation of the image to within a precision of about $0.12^{\circ}$ (Lessard et al. 2001).

The total integrated signal for a shower image (the shower size) is roughly proportional to the energy of the primary $\gamma$-ray. To obtain a better energy estimator, we also correct for the impact parameter of the shower. An approximate measure of the impact parameter of each shower is obtained by measuring the parallax angle between the image centroid (point of maximum shower development) and the source direction. By correcting for the dependence on this centroid distance, one obtains an energy estimator for each candidate $\gamma$-ray event. Monte Carlo simulations of $\gamma$-ray showers are used to determine the relationship between this energy estimator and the actual energy. To determine the energy spectrum for a given data set, a histogram of the energy estimator for candidate $\gamma$-ray events is formed for both the onsource and off-source data. The difference in the on-off histograms is then converted to an energy spectrum by using the effective area function calculated by detailed Monte Carlo simulations of the air shower and detector.

For the present analysis, the spectra were reconstructed using either the method of Mohanty et al. (1998 and Krennrich et al. (2001; method A) or the forward folding method of Rebillot et al. (2006; method B). In method A, the combined effects of the spectral deconvolution (assuming a lognormal resolution function) and energy-dependent efficiency are taken into account by dividing the binned fluxes by the effective area function. The spectral slope is then determined by fitting a spectral model to the unfolded spectral data points by $\chi^{2}$ minimization. Following Krennrich et al. (2001) we assume that the Mrk 421 spectrum can be characterized by a single power law with an exponential energy cutoff at a fixed value of $4.3 \mathrm{TeV}$.

In method B, instead of fitting to the unfolded spectrum, we directly compared the energy estimator distribution of a simulated $\gamma$-ray data set to the measured distribution for the real data. A grid search was used to find the parameters (flux normalization and spectral index) that resulted in the best fit to the data. To make this computationally tractable, we did not repeat the simulations for each trial, but drew our random data sets from a weighted simulation database. The measured and simulated energy-estimator distributions were compared to form a likelihood for each trial value of the spectral index. An adaptive grid search was used to find the best-fit value of the flux normalization and spectral index and to determine the confidence interval on the best-fit parameter.

For our spectral analysis, it is important to know the energy scale to get a reliable value for the flux normalization. For this season the trigger condition (which directly impacts the energy threshold) consisted of the requirement that three adjacent PMT signals exceeded a threshold of $32 \mathrm{mV}$. By comparing muonring images obtained in the data with those generated in Monte Carlo simulations, we obtained a gain calibration for the 2001 season.

Even in its relatively active state, the $\gamma$-ray observations of Mrk 421 are often background limited. To adequately characterize the background of misidentified cosmic-ray events we used either an on-off analysis or a tracking analysis. For these observations, we employed an observing strategy that was a compromise between the desire to obtain continuous coverage for the time-series analysis and the need for interspersed off-source control runs to adequately characterize systematic variations in background.

In a typical night, a single on-off pair was taken in which a 28 minute on-source run with the 10 m telescope tracking Mrk 421 was followed by another 28 minute observation that tracked the same range of azimuth and elevation angles, but offset by $30^{\mathrm{m}}$ in right ascension. This mode of data taking reduces systematic errors from differences in sky brightness, atmospheric conditions, and instrument variations but reduces the duty cycle of observations. Other runs taken in the same night were typically acquired in a "staring" or "tracking" mode where the telescope continuously tracks the source without interruption for off-source observation.

Data selection cuts based on the rms width and length of the shower images were used to determine candidate $\gamma$-ray events. The angle between the major axis of the Cerenkov image and the line connecting the image centroid to the angular position of the source (designated $\alpha$ ) is used to define the signal and background region in the image parameter space. In each on-source run, the events whose major axis points to the position of the source in the field of view are used to estimate the signal, and those misaligned events pointing away from the center are used to determine the background (de la Calle Pérez et al. 2003). The number of candidate $\gamma$-ray events was determined by subtracting the number of on-source events from off-source events. Significances were determined by the method of Li \& Ma (1983).

Since the data were taken over a range of zenith angles, a firstorder correction was made to take into account variations in energy threshold and effective collection area with zenith angle. For the light curves presented in this paper we choose the simple empirical method of normalizing to the flux from the Crab Nebula at the corresponding zenith angle. Like relative photometry, this method cancels systematic errors and should provide a better method for eventually combining data with data obtained by different detectors calibrated by different Monte Carlo simulations. However, this method results in a systematic (second order) error if the Mrk 421 spectrum differs significantly from that of the Crab Nebula.

The Crab Nebula can be used to empirically quantify the combined effect of these changes for a source with the same spectrum $N(E) \sim E^{-2.49}$ (Hillas et al. 1998). Figure 1 shows a fit to the $\mathrm{Crab}$ rate as a function of zenith angle for 50 on-off runs from 2000 October 25 to the end of the 2001 observing season.

The average spectral index of Mrk 421 has been measured to have a similar value to the Crab Nebula in the Whipple energy range, but with evidence for some spectral variability (Piron et al. 2001; Krennrich et al. 2002; Aharonian et al. 2002). Since the energy threshold only varies by a factor of roughly $30 \%$ over the range of zenith angles of our observations, we estimate a systematic error in the flux of roughly $10 \%$ if we assume that the spectral index varies by $\Delta \Gamma \simeq 0.3$. But we lack the ability to determine the spectral index to this precision for most individual runs, and thus subsequently admit the possibility of some systematic errors and normalize our observations to a functional form for the zenithangle-dependent rate from the Crab Nebula:

$$
R_{\mathrm{Crab}} \theta=\frac{7.423 \sec ^{2} \theta}{\left[\exp (0.5 \sec \theta) \sec ^{2} \theta\right]^{1.49}}
$$




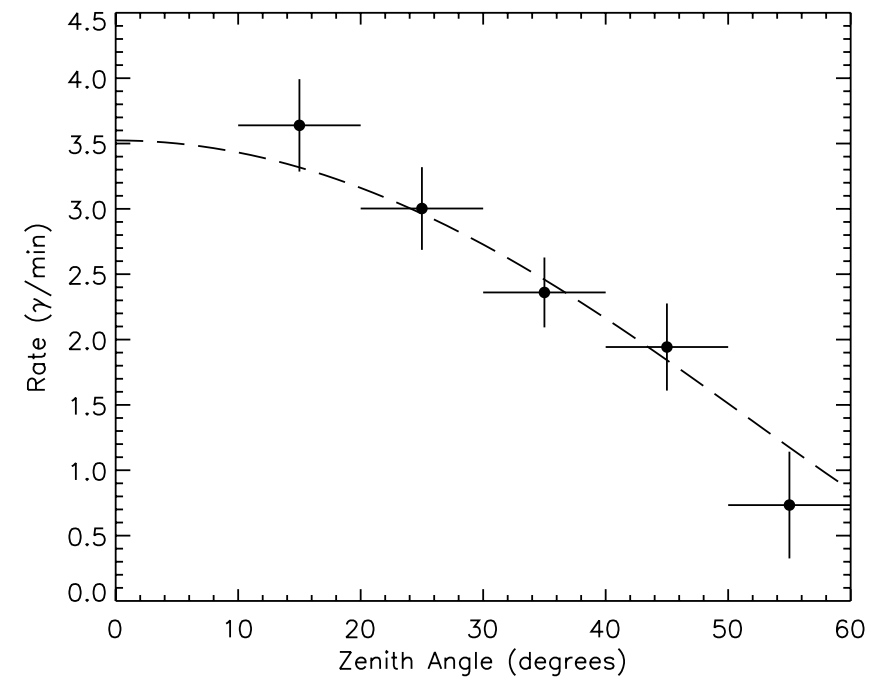

FIG. 1.-Dependence of the observed rate of $\gamma$-rays from the Crab Nebula as a function of zenith angle, as observed in the 2000-2001 season. The fit function indicated by the dashed curve was used to normalize the raw Mrk 421 rates.

This function is derived from an empirical fit to data taken on the Crab Nebula at various zenith angles during the same observing season (see Fig. 1).

Total observations for the 2001 March campaign came to $10.3 \mathrm{hr}$ of on-off data and $37.7 \mathrm{hr}$ of tracking data for a total of $48 \mathrm{hr}$ of data, only a fraction of which are covered in this paper.
For our multiwavelength studies we chose three arbitrary, a priori bin widths: (1) one corresponding to a single day of observations, (2) one corresponding to a single 28 minute data run, and $(3)$ a shorter bin width of $\approx 4$ minutes. The latter choice is arbitrary, but it was derived from past analyses of strong flares as giving a good compromise between statistics and temporal resolution.

\subsubsection{HEGRA Observatory $\gamma$-Ray Data}

The HEGRA TeV data, light curve, and spectra utilized in this paper have been previously published by Aharonian et al. (2002). Please refer to the original paper for details concerning the data reduction.

\subsection{Combining Whipple and HEGRA $\gamma$-Ray Data}

The locations of the Whipple and HEGRA telescopes, in Arizona and the Canary islands, respectively, separated by approximately $6 \mathrm{hr}$, make it possible to achieve uninterrupted coverage of Mrk 421 during the spring, when the target is observable at small zenith angles for up to $7 \mathrm{hr}$ each night from each site. The visibility windows thus complement each other very well (see Figs. 2 and 3 ) but do not overlap. For this campaign we achieved an unprecedented coverage of the target: as illustrated in Tables 2 and 3 the total net exposure time for the two TeV observatories was about $62 \mathrm{hr}$, over seven nights. Ignoring gaps shorter than $1.5 \mathrm{hr}$, the on-source time was of about $72 \mathrm{hr}$, i.e., more than $10 \mathrm{hr}$ per night.

The combination of data from different instruments is always a very delicate step, most robustly addressed by comparing data taken simultaneously, which is not possible for Whipple and HEGRA. The next best option is to cross-calibrate the data using a standard

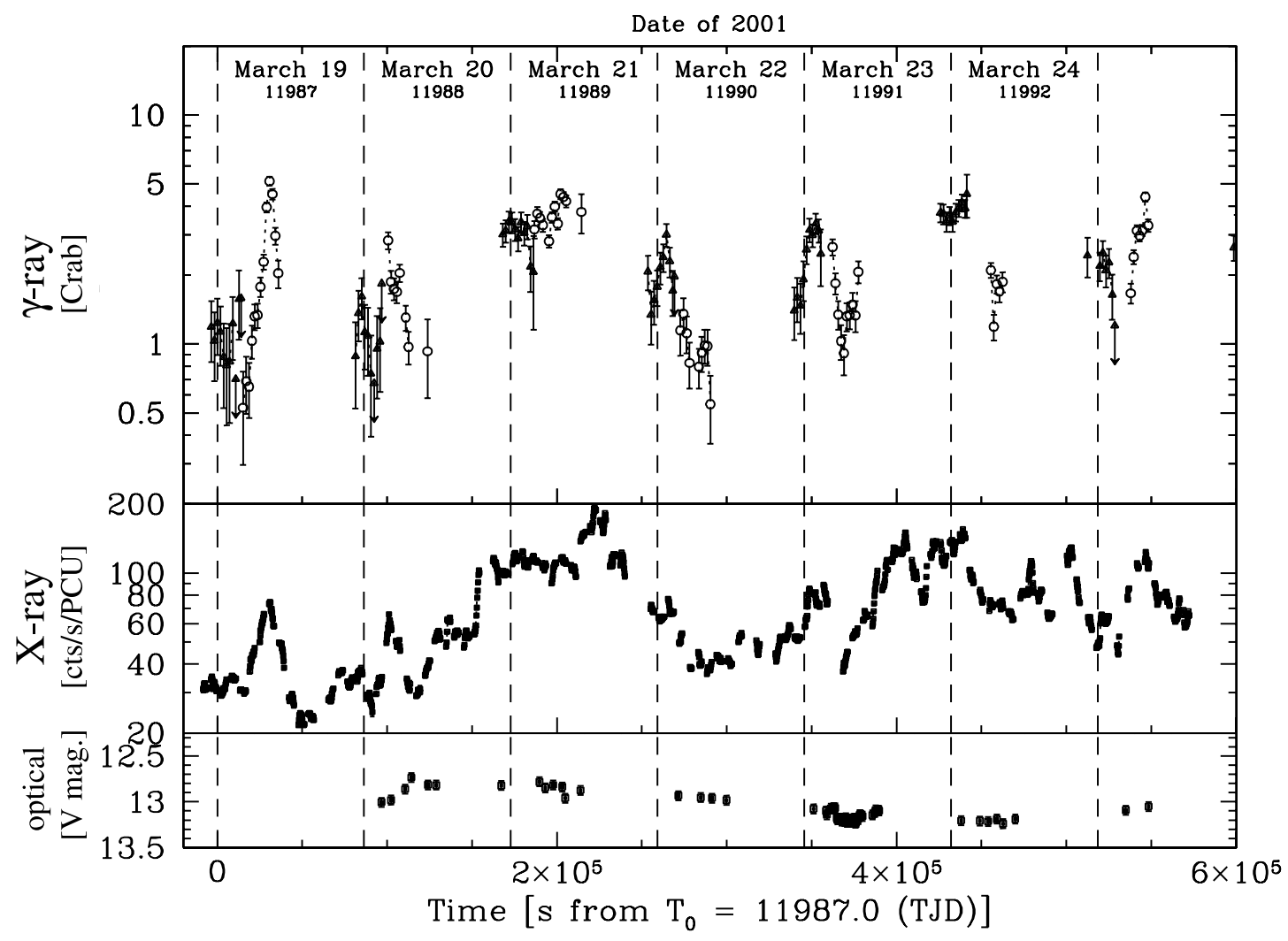

FIG. 2.- Simultaneous optical ( $V$ band, bottom), X-ray $(2-10 \mathrm{keV}$, middle), and TeV $\gamma$-ray $(E>0.4$ TeV, but see $\S 2.3$, top) light curves for Mrk 421 for the March 18-25 period. RXTE PCA data are shown here in $256 \mathrm{~s}$ bins. HEGRA data (black triangles) are integrated over $1800 \mathrm{~s}$ bins, Whipple data (white circles) over $1680 \mathrm{~s}$ bins.

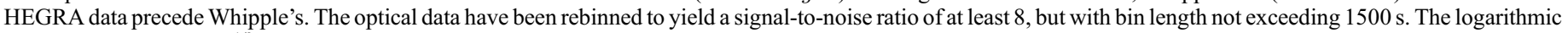

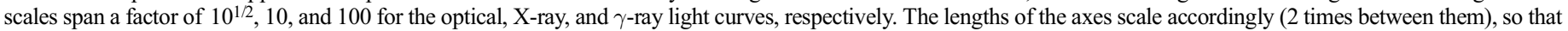
relative amplitude variability can be directly compared. 

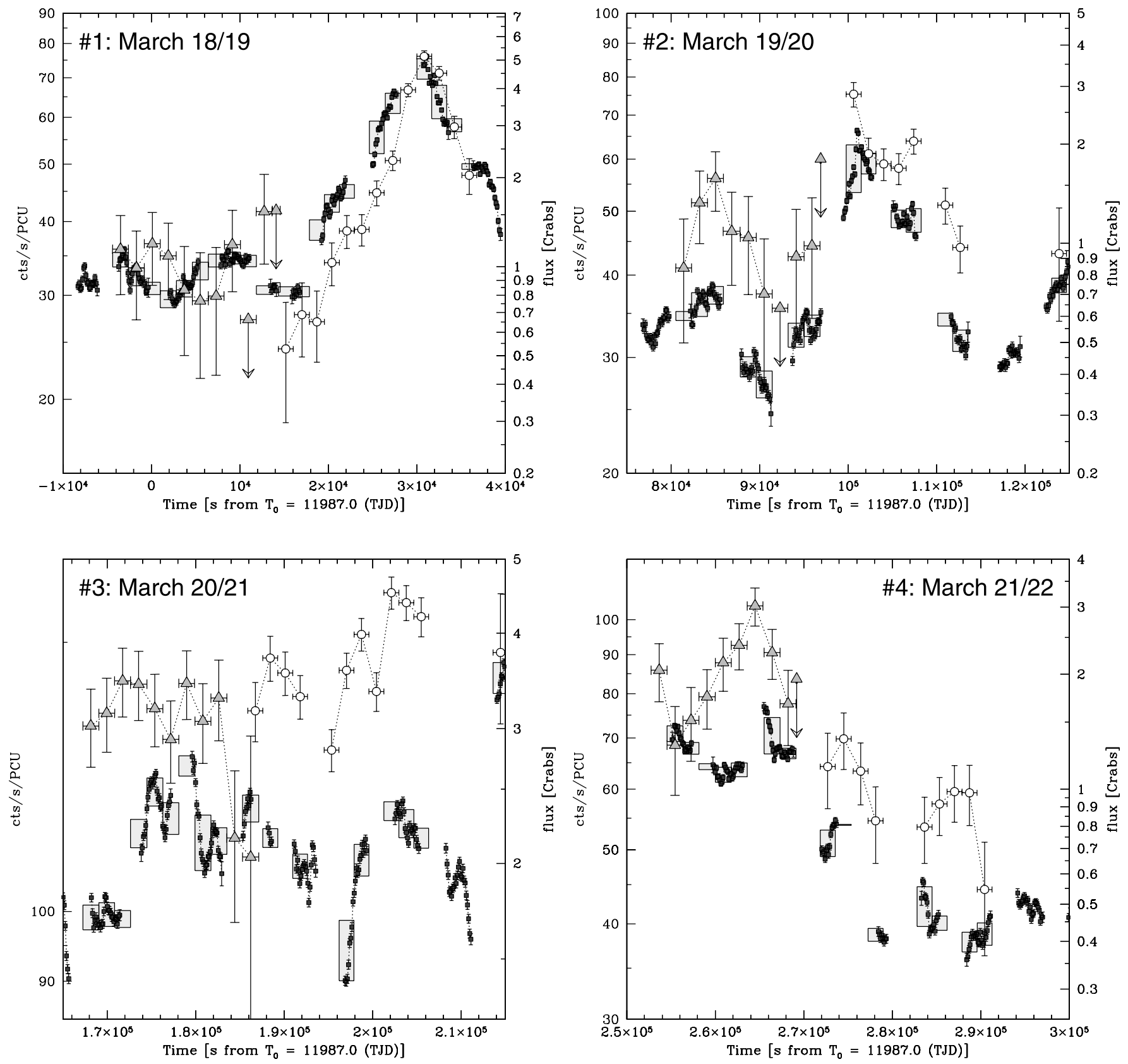

FIG. $3 a$

FIG. 3.-Simultaneous $2-10 \mathrm{keV} \mathrm{X-ray} \mathrm{and} \mathrm{TeV} \mathrm{(see} \mathrm{text)} \gamma$-ray light curves for individual nights. Light gray triangles show HEGRA data, in $\approx 1800 \mathrm{~s}$ bins; white circles show Whipple data, integrated over $\approx 1680 \mathrm{~s}$ bins. Dense dark gray points show $R X T E$ PCA data, in $128 \mathrm{~s}$ bins. The shaded boxes represent the average and variance of the X-ray data for each (longer duration) $\mathrm{TeV}$ bin, which are the values used in the flux-flux correlation analyses. The rate scales for the X-ray data are on the left $Y$-axes, and the flux scales for the TeV data on the right $Y$-axes. The time span is the same for all panels, $50 \mathrm{ks}$. The vertical scales are not the same in all panels, but are adjusted to show each day in the best possible detail. The X-ray dynamic ranges are (time ordered ) 6, 5,2, 4, 4, 4, and 4 times. In order to allow for an easier comparison of the relative variability amplitude, in all panels the $Y$-axis range for the $\gamma$-ray light curve is the square of that used to plot the X-ray data. The source shows strong, highly correlated variability in both energy bands, with no evidence for any interband lag (but see $\S 3.3$ ).

candle as a reference, typically the Crab Nebula for high-energy emission. The main issue concerning the combination of the data from the Whipple and HEGRA telescopes arises from the fact that (1) they gather data with different lower energy thresholds, 0.4 and $1.0 \mathrm{TeV}$, respectively, and that (2) the Mrk $421 \mathrm{TeV}$ spectrum is in general (significantly) harder than the Crab's. To illustrate the problem, let us take spectral indices $\Gamma=2.5$ for the Crab and $\Gamma=$ 2.2 for Mrk 421 (Krennrich et al. 2002; Aharonian et al. 2002). If we ignore the fact that a detector response is energy dependent we can compare the ratio between the Mrk 421 and Crab fluxes computed for different energy thresholds, namely, 0.4 and $1 \mathrm{TeV}$. The flux above an energy $E$ for a simple power law with spectral index $\Gamma$ is

$$
F(E, \Gamma)=\frac{N_{0} E^{1-\Gamma}}{\Gamma-1} .
$$



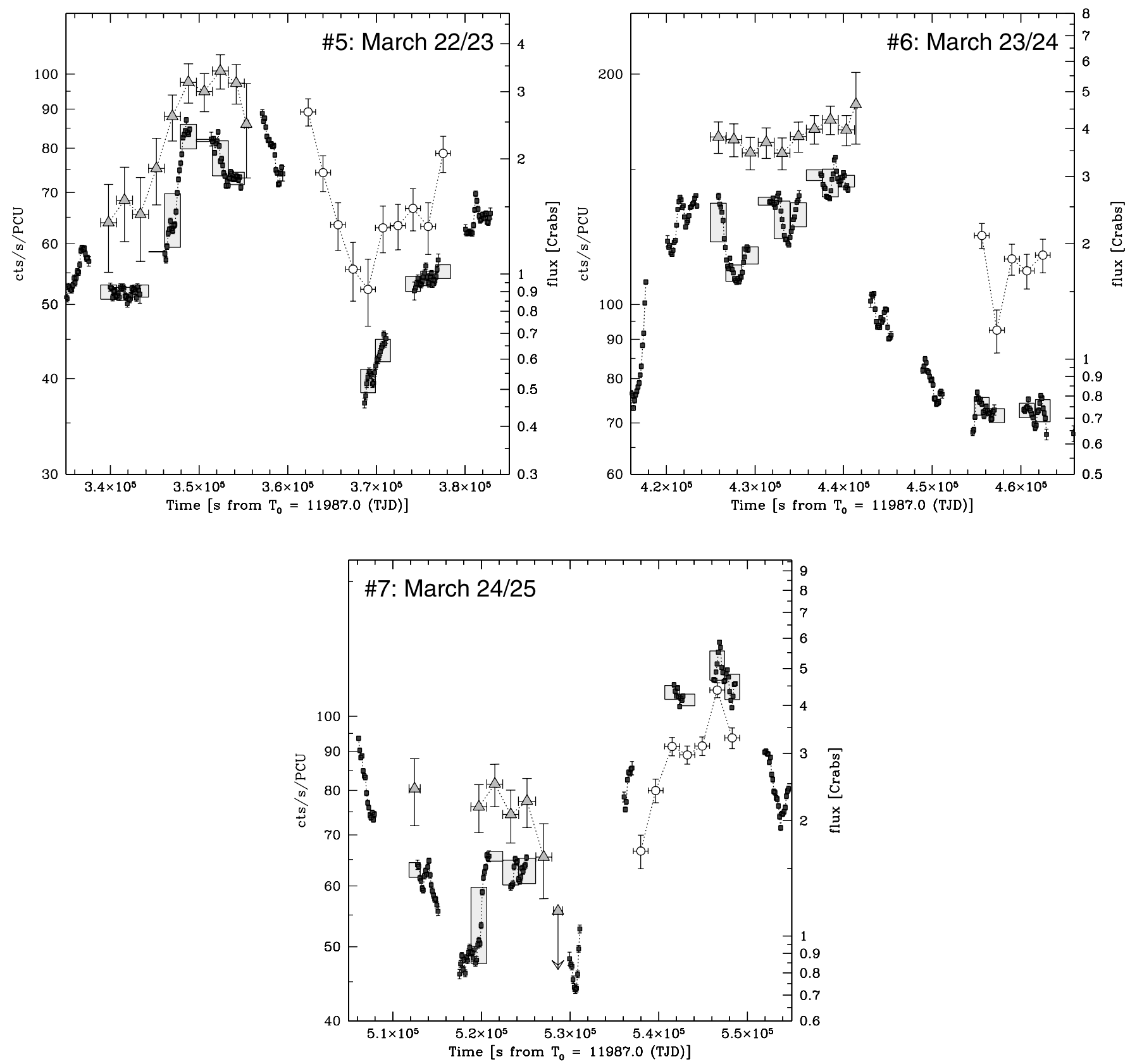

FIG. $3 b$

Therefore

$$
\begin{aligned}
\tilde{F}_{\text {Mrk 421 }}(E) & =\frac{F\left(E, \Gamma_{\text {Mrk } 421}\right)}{F\left(E, \Gamma_{\text {Crab }}\right)} \\
& =\frac{N_{0, \text { Mrk 421 }}}{N_{0, \text { Crab }}} \frac{\Gamma_{\text {Crab }}-1}{\Gamma_{\text {Mrk 421 }}-1} E^{\Gamma_{\text {Crab }}-\Gamma_{\text {Mrk } 421}},
\end{aligned}
$$

and comparing data taken at two different thresholds yields

$$
f=\frac{\tilde{F}_{\text {Mrk 421 }}\left(E_{A}\right)}{\tilde{F}_{\text {Mrk 421 }}\left(E_{B}\right)}=\left(\frac{E_{A}}{E_{B}}\right)^{\Gamma_{\text {Crab }}-\Gamma_{\text {Mrk 421 }}} .
$$

For $E_{A}=1.0 \mathrm{TeV}$ (HEGRA) and $E_{B}=0.4 \mathrm{TeV}$ (Whipple), this ratio is 1.32 ; i.e., a spectrum yielding a Whipple flux of 1 crab will be observed at 1.32 crab by HEGRA. Moreover, this effect is a function of the spectral indices. While we can safely consider the Crab spectrum nonvariable, significant variability is observed in Mrk 421 (Krennrich et al. 2002; A haronian et al. 2002). The value of $f=1.32$ for the HEGRA/Whipple flux ratio obtained for $\Gamma_{\text {Mrk } 421}=2.2$ becomes $f=1.44,1.10$, and 0.83 for $\Gamma_{\text {Mrk } 421}=$ 2.1, 2.4, and 2.7, respectively. Hence, HEGRA data would also show a larger variance. Finally, it is worth noting that the ratios between the 2001 March Whipple and HEGRA mean fluxes and between their variances are consistent with what is expected based on the simple arguments just illustrated and the observed spectral indices.

Since it is not possible to derive from first principles a robust way to convert Whipple and HEGRA light-curve measurements into each other, we conclude that the most robust procedure at 
TABLE 2

$R X T E$ PCA and Whipple/HEGRA Overlap Statistics

\begin{tabular}{|c|c|c|c|c|c|c|c|c|}
\hline $\begin{array}{l}\text { NIGHT } \\
(1)\end{array}$ & $\begin{array}{c}\text { Date }^{a} \\
\text { (2) }\end{array}$ & $\begin{array}{c}\text { Date } \\
\text { (MJD) } \\
(3)\end{array}$ & $\begin{array}{c}\text { WhIPPLE EXP. TIME } \\
\text { (4) }\end{array}$ & \multicolumn{2}{|c|}{ Overlap Fraction } & $\begin{array}{l}\text { HEGRA Exp. TIME } \\
\text { (7) }\end{array}$ & \multicolumn{2}{|c|}{ Overlap Fraction } \\
\hline 1 & Mar 18/19 & $51986 / 51987$ & $6 \mathrm{hr} 4 \mathrm{~min}$ & 42 & $11 / 13(85 \%)$ & $5 \mathrm{hr} 14 \mathrm{~min}$ & 55 & $11 / 11(100 \%)$ \\
\hline 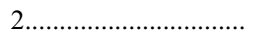 & Mar 19/20 & $51987 / 51988$ & $3 \mathrm{hr} 44 \mathrm{~min}$ & 67 & $7 / 8(87 \%)$ & $4 \mathrm{hr} 33 \mathrm{~min}$ & 60 & $8 / 10(80 \%)$ \\
\hline 3.................................. & Mar 20/21 & $51988 / 51989$ & $5 \mathrm{hr} 36 \mathrm{~min}$ & 45 & $9 / 12(75 \%)$ & $5 \mathrm{hr} 30 \mathrm{~min}$ & 54 & $10 / 11(91 \%)$ \\
\hline 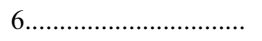 & Mar 23/24 & $51991 / 51992$ & $2 \mathrm{hr} 20 \mathrm{~min}$ & 55 & $4 / 5(80 \%)$ & $4 \mathrm{hr} 35 \mathrm{~min}$ & 58 & 9/10 (90\%) \\
\hline 7............................... & Mar 24/25 & $51992 / 51993$ & $3 \mathrm{hr} 16 \mathrm{~min}$ & 31 & $4 / 7(57 \%)$ & $3 \mathrm{hr} 10 \mathrm{~min}$ & 37 & $5 / 7(71 \%)$ \\
\hline All …........................ & Mar $18-25$ & $51986 / 51993$ & $29 \mathrm{hr} 52 \mathrm{~min}$ & 44 & $47 / 64(73 \%)$ & $32 \mathrm{hr} 14 \mathrm{~min}$ & 54 & $58 / 69(84 \%)$ \\
\hline
\end{tabular}

${ }^{a}$ HEGRA observing windows typically extend across UTC midnight. All the Whipple observing windows occur after the UTC midnight.

${ }^{\mathrm{b}}$ Ratio between the actual RXTE PCA on-source time during Whipple (HEGRA) runs and the actual total Whipple (HEGRA) observing time.

${ }^{c}$ Fraction of Whipple (HEGRA) $\approx 0.5 \mathrm{hr}$ runs with some overlapping $R X T E$ PCA data. The overlap fraction ranges between $7 \%$ and $100 \%$, averaging to the number reported in cols. (5) and (8).

hand is to scale the data of one telescope to the same mean and variance of those of the other one. We adopt Whipple as a reference and scale the HEGRA fluxes. We deem this approach to be reliable, primarily because of the large size of the two data sets: they both span 7 days (with alternating visibility windows) and have comparable variability amplitudes; thus, both should be sampling an equally representative subset of the source phenomenology. Being a linear conversion, this procedure cannot correct for the slight nonlinearity of the Whipple versus HEGRA flux relationship introduced by the brightness-hardness correlation of the spectra. In order to mitigate the effect of outliers, and because of the intrinsic exponential nature of the source variability, we performed the scaling of mean and variance on the logarithm of the light curves.

\subsection{Optical Data}

UBVRI optical monitoring was performed using the HarvardSmithsonian 48 inch telescope on Mt. Hopkins. Data were analyzed using relative aperture photometry, using comparison star No. 3 as listed in Villata et al. (1998). Galaxy background light was subtracted using the simple empirical method described in Nilsson et al. (1999). Here we use their determination of the contribution of Mrk 421 galaxy background light in the $R$ band but extrapolate this to the $V$ band using the $R-V$ color of the host galaxy as given in Hickson et al. (1982). The errors shown on the light curve are the systematic uncertainties, which dominate the small statistical errors. These error bars were determined from the measured variance of the reference stars with respect to each other and may not include other effects such as the bleeding of starlight from the bright stars SAO 62387 and 62392 that lie about $2^{\prime}$ from Mrk 421, in the 48 inch telescope field of view. Other systematic effects come from the relatively high level of galaxy light from Mrk 421 and the very nearby satellite elliptical galaxy. These problems, coupled with the lack of good reference stars, combine to make Mrk 421 one of the more difficult BL Lac objects for optical monitoring; we estimate that this measurement should be given a systematic flux uncertainty of about $15 \%$.

\section{TEMPORAL ANALYSIS \\ 3.1. RXTE/Whipple + HEGRA Data Overlap}

The principal statistics regarding the quality of the X-ray $/ \gamma$-ray overlap are summarized in Tables 2 and 3. There we report two different measures of the overlap between the two telescopes. The fractions listed in columns (5) and (8) refer to the absolute covering factor by RXTE of the on-source time of Whipple or HEGRA.

TABLE 3

$R X T E$ PCA AND WhIPPLE + HEGRA OVERLAP STATISTICS

\begin{tabular}{|c|c|c|c|c|c|}
\hline \multirow[b]{2}{*}{$\begin{array}{l}\text { NIGHT } \\
(1)\end{array}$} & \multirow[b]{2}{*}{$\begin{array}{l}\text { DAtE }^{a} \\
(2)\end{array}$} & \multirow[b]{2}{*}{$\begin{array}{c}\text { Date } \\
\text { (MJD) } \\
(3)\end{array}$} & \multirow[b]{2}{*}{$\begin{array}{c}\text { TeV Exp. Time } \\
\text { (4) }\end{array}$} & \multicolumn{2}{|c|}{ Overlap Fraction } \\
\hline & & & & $\begin{array}{c}\text { Detailed }^{\mathrm{b}} \\
(\%) \\
(5)\end{array}$ & $\begin{array}{c}\text { Run-by-Run }{ }^{c} \\
(6)\end{array}$ \\
\hline 1 & Mar 18/19 & $51986 / 51987$ & $11 \mathrm{hr} 18 \mathrm{~min}$ & 48 & $22 / 24(92 \%)$ \\
\hline 2 & Mar 19/20 & $51987 / 51988$ & 8 hr 17 min & 63 & $15 / 18(83 \%)$ \\
\hline 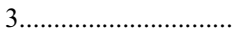 & Mar 20/21 & $51988 / 51989$ & $11 \mathrm{hr} 6 \mathrm{~min}$ & 48 & $18 / 23(78 \%)$ \\
\hline $4 \ldots \ldots \ldots \ldots \ldots \ldots \ldots \ldots$ & Mar 21/22 & $51989 / 51990$ & $8 \mathrm{hr} 44 \mathrm{~min}$ & 49 & $14 / 19(74 \%)$ \\
\hline $5 \ldots \ldots \ldots \ldots \ldots \ldots \ldots \ldots .$. & Mar 22/23 & $51990 / 51991$ & $9 \mathrm{hr} 17 \mathrm{~min}$ & 43 & $13 / 20(65 \%)$ \\
\hline 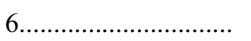 & Mar 23/24 & $51991 / 51992$ & $6 \mathrm{hr} 55 \mathrm{~min}$ & 57 & $13 / 15(87 \%)$ \\
\hline 7............................ & Mar 24/25 & $51992 / 51993$ & $6 \mathrm{hr} 26 \mathrm{~min}$ & 34 & 9/14 (64\%) \\
\hline All .............................. & Mar 18-25 & $51986 / 51993$ & $62 \mathrm{hr} 6 \mathrm{~min}$ & 49 & $104 / 133(78 \%)$ \\
\hline
\end{tabular}



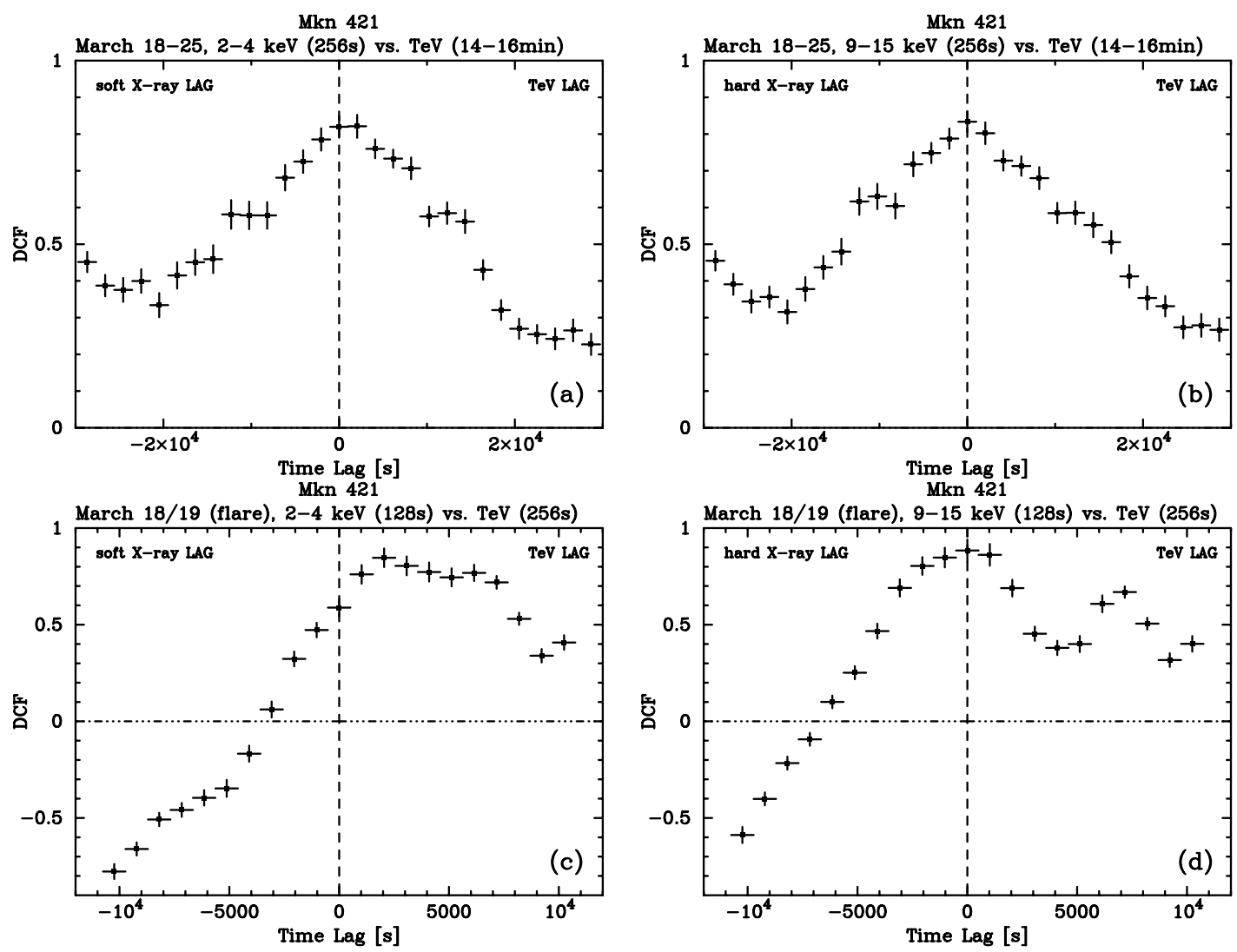

FIG. 4. - Cross-correlation between the X-ray and the TeV light curves: (a) 2-4 keV vs. TeV (Whipple + HEGRA) for the whole campaign (computed over $2048 \mathrm{~s}$ bins, from X-ray data on $256 \mathrm{~s}$ bins, and $\mathrm{TeV}$ data on $\simeq 750-900 \mathrm{~s}$ bins); (b) $9-15 \mathrm{keV}$ vs. TeV (Whipple + HEGRA) for the whole campaign (computed over $2048 \mathrm{~s}$ bins, from $\mathrm{X}$-ray data on $256 \mathrm{~s}$ bins, and $\mathrm{TeV}$ data on $\simeq 750-900 \mathrm{~s}$ bins); (c) $2-4 \mathrm{keV}$ vs. TeV (Whipple) for the night of March $18-19$ (the flare of Fig. $3 a$, top left, computed over $1024 \mathrm{~s}$ bins, from X-ray data on $128 \mathrm{~s}$ bins, and Whipple data on $256 \mathrm{~s}$ bins); (d) $9-15 \mathrm{keV}$ vs. TeV (Whipple) for the night of March $18-19$ (computed over $1024 \mathrm{~s}$ bins, from $\mathrm{X}$-ray data on $128 \mathrm{~s}$ bins, and Whipple data on $256 \mathrm{~s}$ bins).

These numbers give an idea of how representative the collected X-ray data are of the actual X-ray brightness. In column (6) (and col. [9]), we report the fraction of Whipple (and HEGRA) data points (from the run-by-run light curves) that have a nonzero overlap with any fraction of the $R X T E$ PCA GTIs: the actual exposed fractions for the individual runs range between $7 \%$ and $100 \%$ (with averages of the order of the value reported in cols. [5] and [8]). Although the RXTE schedule was optimized to observe Mrk 421 as much as possible, ${ }^{21}$ we still missed about one-fourth of the Whipple runs, and during each Whipple/HEGRA $\approx 0.5 \mathrm{hr}$ integration window X-ray data were collected only for about $50 \%$ of the time. Since the X-ray and $\gamma$-ray brightness can vary significantly on timescales faster than $\approx 0.5 \mathrm{hr}$, we may expect this to affect the X-ray/TeV flux correlation. We carefully inspected the X-ray light curves to assess the impact of the uneven coverage and concluded that the effect is not important. The largest X-ray variation during any $0.5 \mathrm{hr}$ interval is $\sim 30 \%$, with an average of $\sim 15 \%$.

\subsection{Light Curves}

The resulting light curves for the full week are shown in Figure 2. The overall brightness ranges are approximately $0.5,10$, and 10 times for optical, X-rays, and $\gamma$-rays, respectively. Although the time coverage was the best possible and the data sets encompass several days and multiple flares, these ranges can be

\footnotetext{
21 Between Match 19 and 25 RXTE observed Mrk 421 for 94 of 102 orbits, and in only one case were there two consecutive orbits missed. One missed orbit corresponds to $\mathrm{a} \simeq 8.5 \mathrm{ks}$ gap.
}

affected by the lack of continuous coverage by the ground-based telescopes (e.g., there are no optical or $\gamma$-ray data for the time of the lowest X-ray flux, on March 19).

Although the large gaps in the $\gamma$-ray light curves may limit the interpretation, some effects are obvious. The source shows strong variations at both bands, and these variations are highly correlated, with features in one band generally showing up in the other band as well. This is clearly illustrated in Figure 3, where we plot $50 \mathrm{ks}$ sections of each night, centered on the time window covered by the TeV observations.

The best example is the March 19 observation, when a welldefined, isolated flare was observed both in the X-ray and $\gamma$-ray bands, from its onset through its peak (more in $\S \S 3.3$ and 3.4). In several other nights, despite the lack of other major isolated features in the light curve(s) that can provide an unambiguous reference for comparison, the variations at X-ray and $\gamma$-ray are clearly correlated. Given the high degree of variability (rapid and high amplitude in both bands) it is always possible that intrinsically uncorrelated flares in the two bands end up being simultaneous by chance. However, the unprecedented level of detail (i.e., time resolution) and extension of the simultaneous coverage allow us to match several relatively minor features in the light curves and effectively confirm the correlation of the variations in the X-ray and $\gamma$-ray bands.

In particular, within the detail allowed by the coarser TeV sampling, the X-ray and $\gamma$-ray light curves seem to track each other closely in all cases when the sampling is good, namely, for nights 1 , $2,4,5$, and 7 . For nights 3 and 6 there seem to be significant deviations from this general trend. It is, however, worth noting that 

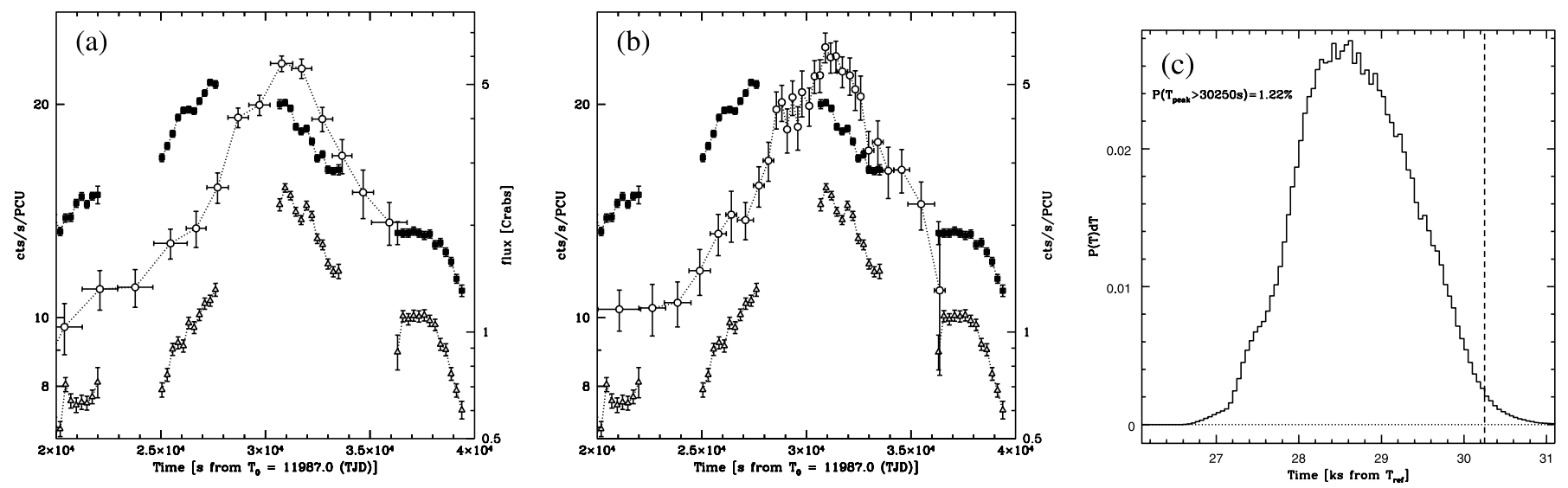

FIG. 5.- (a) March 19 light curves for the Whipple $\gamma$-ray band (connected white circles, $\simeq 1000$ or $1680 \mathrm{~s}$ bins) and two X-ray bands, $2-4$ keV (black squares) and $9-15$ keV (light gray triangles), both in $256 \mathrm{~s}$ bins. The rate scale for the X-ray data is on the left $Y$-axis, and the flux scale for the Whipple data on the right $Y$-axis. To allow for an easier comparison of the relative variability amplitude, the $Y$-axis range for the $\gamma$-ray light curve (16 times) is the square of that used to plot the X-ray data (4 times). (b) Same as $(a)$, except that here the TeV ( Whipple) light-curve data have been adaptively rebinned from the $256 \mathrm{~s}$ data to a signal-to-noise ratio of at least 6 . ( $c$ ) Probability distribution of soft X-ray flare peak time derived from the general statistical properties of the short-term variability (see $\S 3.3$ ). The vertical dashed line marks the leftmost boundary of the Whipple time interval comprising the TeV flare peak.

these two nights correspond to the highest brightness level in the $\mathrm{X}$-ray, and the X-ray light curves show several fast variations (intraorbit) that may not have been sampled properly by the $\mathrm{TeV}$ observations (e.g., in 3), or may in fact not have a TeV counterpart (e.g., night 6, where the TeV data seem to have good signalto-noise ratio). In a broad sense, even in nights 3 and 6 , the data are consistent with correlated variations in the two energy bands.

In the next two subsections ( $\S \S 3.3$ and 3.4$)$, we examine in detail the properties of the X-ray/TeV correlation from two complementary points of view, phase and amplitude.

\subsection{X-Ray/TeV Interband Lags}

Cross-correlation functions were measured to quantify the degree of correlation and phase differences (lags) between variations in the X-ray and $\gamma$-ray bands, using the discrete correlation function (DCF) of Edelson \& Krolik (1988). For the X-rays we consider two different bands (2-4 and 9-15 keV) at the usable ends of the PCA bandpass, to explore the possible energy dependence of the correlation. The results for the whole week-long and March 19 flare data are shown in Figures $4 a-4 d$. It is worth noting that, as discussed in F08, variations in the harder X-ray emission seem overall to lag those in the softer PCA band.

\subsubsection{Full Week-long Data Set}

For what concerns the full week data set (Figs. $4 a$ and $4 b$ ), we do not find a measurable lag, with either X-ray band. The statistical properties of the DCF are complicated by the presence of several regular patterns in the data trains (chiefly the diurnal gaps in the ground-based $\mathrm{TeV}$ data and the orbital gaps of the $R X T E$ data). The DCFs peak at zero lag, and the correlation coefficients are quite high, $\simeq 0.8$, despite the poor sampling (the combination of the large diurnal gaps due to the ground-based visibility and the $R X T E$ orbital gaps yields an efficiency of about $\frac{1}{2} \times \frac{3}{4} \simeq 40 \%$ ). By means of simple data-based simulations comprising flux randomization (e.g., Peterson et al. 1998), and the effect of introducing a shift in one of the time series, we estimate an upper limit of $|\tau| \lesssim 3 \mathrm{ks}$ on the value of the soft X-ray/ $\gamma$-ray lag, possibly smaller for the harder X-ray $/ \gamma$-ray case.

\subsubsection{The March 19 Flare}

Besides the full data set, we focused our attention on the isolated outburst of March 18/19 that uniquely comprises many fa- vorable observational characteristics, namely, (1) the best TeV coverage, (2) the fewest $R X T E$ data gaps, (3) the best $R X T E /$ Whipple overlap, and (4) the largest brightness excursion (in both bands, 10 times in $\gamma$-rays, 3 times in X-rays). Because of this indeed rare combination of properties, the March 18/19 flare's DCF is probably not significantly affected by the sampling. Because of its relative isolation from other outbursts and its large amplitude, we can regard this event as a rather clean "experiment," providing us a good view of the variability mechanism at work. On the other hand, results obtained for this particular event may not necessarily be representative of all flares.

In this case we used the Whipple data in their shortest available time binning, $256 \mathrm{~s}$ bins (an example of this is the peak region of the March 19 burst shown in Fig. 5c). The DCFs for the two different X-ray bands, computed over $1024 \mathrm{~s}$ time steps, are plotted in Figures $4 c$ and $4 d$. The DCFs for both X-ray bands show a high correlation coefficient, peaking at 0.84 (at a lag $\tau \approx+2 \mathrm{ks}$ ) for the soft X-rays, and at 0.88 (at zero lag) for the harder X-rays.

The most remarkable feature is that there seems to be a hint for the $\gamma$-rays lagging the softer X-rays, while being synchronized with the harder X-ray photons. A thorough analysis and characterization of the properties of the X-ray variability is discussed in F08. In Figures $5 a-5 c$, we just show a 20 ks section of the three light curves for March 19, centered on the possible peak position. Although the peak of the outburst was not directly observed in $\mathrm{X}$-rays, we can make the following heuristic arguments concerning the possibility, and value, of an interband lag between the softer X-rays and the $\mathrm{TeV}$ data.

\subsubsection{Constraining the Lag for the March 19 Flare}

By exploiting the statistical knowledge of the characteristics of the X-ray variability during this campaign (see F08), we can try to assess the probability that a lag at the flare peak in fact exists between the $\mathrm{TeV}$ and the soft $\mathrm{X}$-ray light curves. The idea is to assign a probability distribution for the soft X-ray peak to occur at times $t_{\text {peak }}$ during the data gap and then use it to evaluate the probability that the peak in fact occurred within the time interval comprising the peak of the $\mathrm{TeV}$ outburst, which is at $T-T_{\text {ref }} \geq 30.25 \mathrm{ks}$.

The basic building blocks for these probability estimates $\left\{P\left(t_{\text {peak }}\right)\right\}$ are the observed distributions of doubling and halving times, $P\left(\tau_{2}\right)$ and $P\left(\tau_{1 / 2}\right)$, derived from the entire week-long soft 

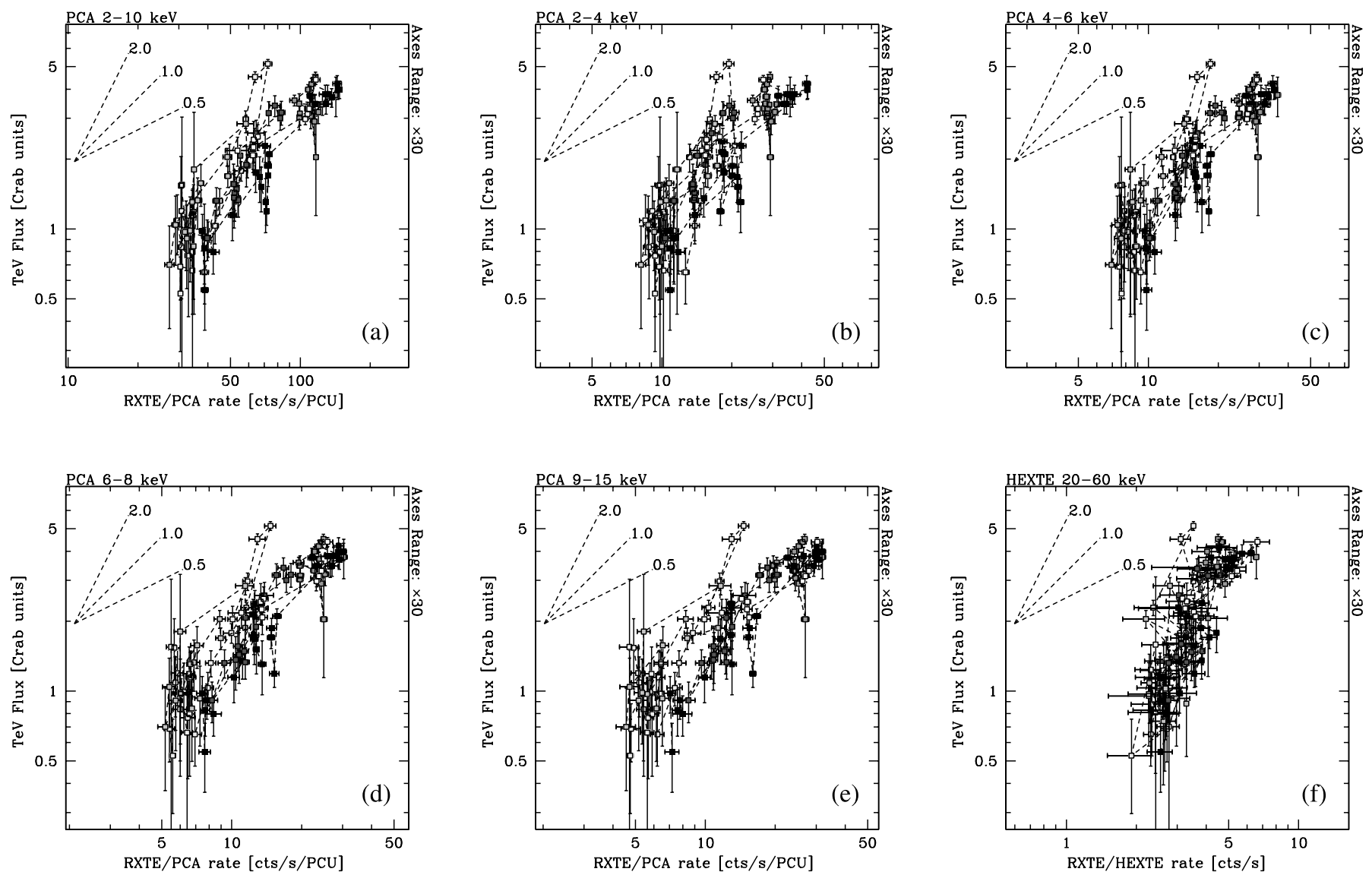

FIG. 6.-Plot of the Whipple + HEGRATeV flux vs. the X-ray count rate in different energy bands (see labels), for the entire week. Different gray shades correspond to different nights. For reference, in each panel are shown segments indicating different slopes for the relationship between the plotted fluxes.

X-ray data set. With these we assign the probability of the peak of the X-ray light curve occurring at a certain time and brightness $\left(t_{\text {peak }}, F_{\text {peak }}\right)$ within the data gap, by taking the joint probability of having the $\tau_{2}$ and $\tau_{1 / 2}$ required to reach each trial $\left(t_{\text {peak }}, F_{\text {peak }}\right)$ position "moving" from the left [i.e., before, $\left.\left(t_{\mathrm{bp}}, F_{\mathrm{bp}}\right)\right]$ and right boundaries of the data gap [i.e., after, $\left.\left(t_{\text {ap }}, F_{\text {ap }}\right)\right]$ :

$$
\begin{aligned}
& P\left(t_{\text {peak }}, F_{\text {peak }}\right) \\
& \sim P\left(\tau_{2}\left(t_{\text {bp }}, F_{\text {bp }} ; t_{\text {peak }}, F_{\text {peak }}\right)\right) P\left(\tau_{1 / 2}\left(t_{\text {ap }}, F_{\text {ap }} ; t_{\text {peak }}, F_{\text {peak }}\right)\right) .
\end{aligned}
$$

Since we are not interested in $F_{\text {peak }}$ this distribution is then summed over all $F_{\text {peak }}$ to yield just $P\left(t_{\text {peak }}\right)$.

The $P\left(\tau_{2}\right)$ and $P\left(\tau_{1 / 2}\right)$ adopted in this analysis were derived from the doubling and halving times from all data pairs whose separation in time $\Delta T_{i j}$ is between 0.25 and $5 \mathrm{ks}$. We restricted our sampling to this subset of data pairs because we wanted the distribution to be representative of the same type of variations that could have occurred during the data gap, which spans $\simeq 3 \mathrm{ks}$. The inclusion of larger pair separations would spuriously bias the probability toward large values of $\tau_{2}$ and $\tau_{1 / 2}$. On the other hand, relaxing the limit on the minimum time separation picks up very fast variations, which are not relevant for this analysis, because their influence on where the peak could fall is marginal (given their limited amplitude), and their overall contribution is already taken into account (smoothed out) by the slopes measured on longer timescales.

We have performed this analysis with different choices of (1) the allowed range of $\Delta T_{i j}$ and (2) the "starting" points on both sides of the gap (namely, we checked points up to $\pm 2 \mathrm{ks}$ from the gap). The results do not change significantly.

The probability distribution for $t_{\text {peak }}$ resulting from this analysis is shown in Figure $5 c$. The average of all the different tests yields a probability of $P\left(t_{\text {peak }}>30.25\right) \simeq 1 \%-2 \%$ for the flare peak to occur later than $T-T_{\text {ref }}=30.25 \mathrm{ks}$, i.e., within the Whipple peak time interval. The most probable $t_{\text {peak }}$ estimated by this method is $t_{\text {peak }}=28.6 \pm 0.8 \mathrm{ks}(1 \sigma), 2 \sigma$ below the first possible time for the peak of the $\mathrm{TeV}$ flare.

We can push this type of analysis a little further to estimate the most likely value for the lag between soft X-rays and TeV. In order to do this we need to assign a probability for the time of the $\mathrm{TeV}$ peak $P\left(t_{\text {peak, TeV }}\right)$. We tried the following simple distributions for $P\left(t_{\text {peak }}, \mathrm{TeV}\right)$ : (1) a uniform distribution within the $1680 \mathrm{~s}$ integration window, (2) a tent function centered on the top interval and going to zero at its boundaries, and (3) a tent function centered on the top interval, but extending over half of each of the two adjacent intervals (i.e., $T-T_{\text {ref }} \simeq 29.7-31.7 \mathrm{ks}$ ). The convolution of the $P\left(t_{\text {peak }, \mathrm{X}}\right)$ with $P\left(t_{\text {peak, TeV }}\right)$ shifted by $\tau$ gives the probability for a given lag $\tau$. The result does not change significantly with the different choices $1-3$, and it is $\tau=2.06_{-0.79}^{+0.69} \mathrm{ks}(1 \sigma)$.

It is important to stress that this analysis rests on a few assumptions that we deem reasonable; we summarize them here.

1. The statistical properties of the X-ray variability change on a timescale longer than our experiment. In this respect we checked that the distribution of $\tau_{2}$ and $\tau_{1 / 2}$ for different subsets of the weeklong data set are consistent with each other.

2. The power spectrum of the variations in $\mathrm{X}$-rays and $\mathrm{TeV}$ is such that the there is only a negligible probability that the peak of 
TABLE 4

Slope of the TeV/X-Ray Correlation

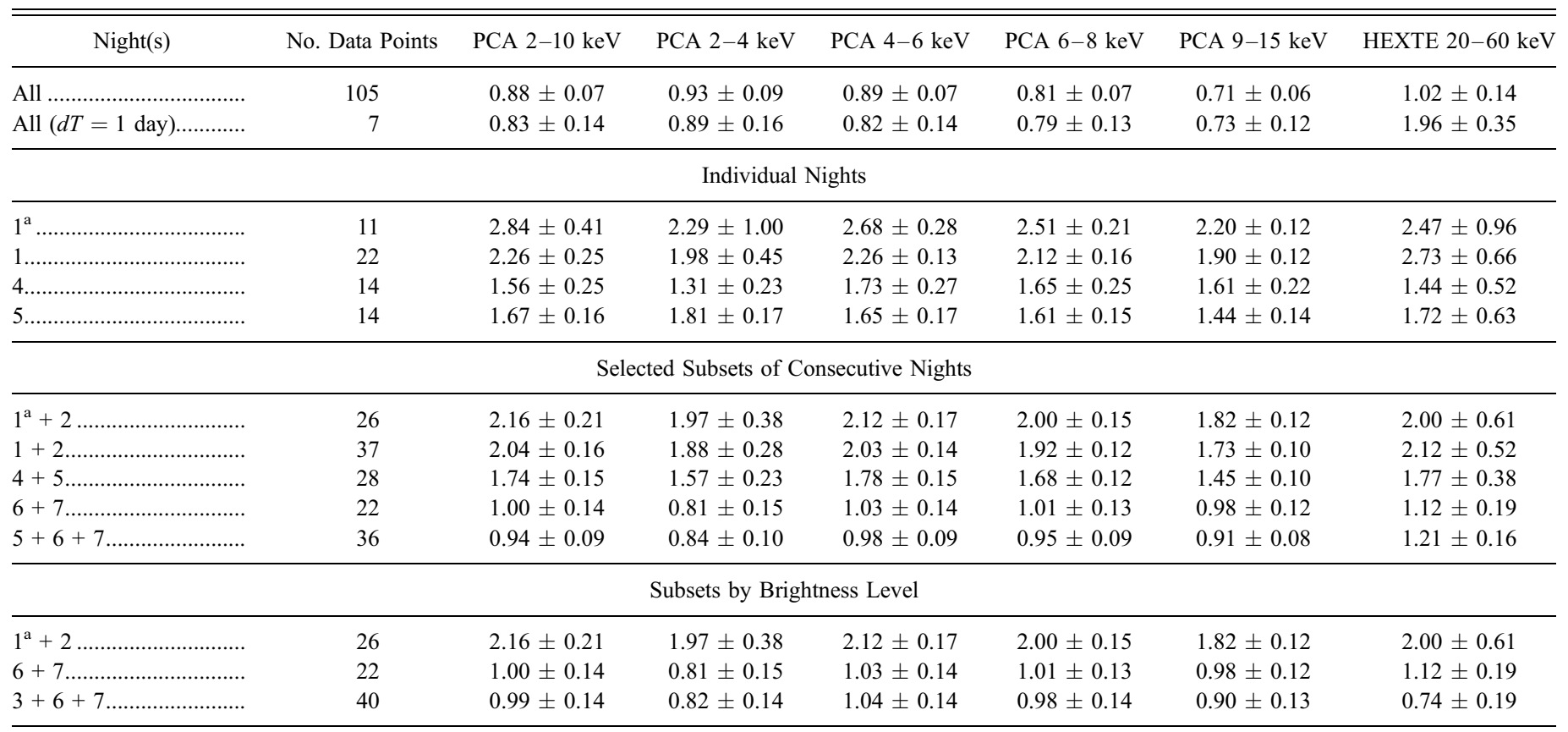

${ }^{\mathrm{a}}$ Whipple (flare) only.

the X-ray light curve occurred before or after the data gap, and that of the $\gamma$-ray light curve during one of the earlier or later integration windows. For instance, we rule out the possibility that the $\mathrm{TeV}$ flare peak could have been reached by means of a very fast and very large amplitude variation (a spike not resolved, and smoothed out, by the coarse Whipple binning), during one of the two Whipple bins falling during the gap in the RXTE data. Moreover, higher sampling Whipple light curves (see Fig. $5 b$ ) provide a further constraint on the probability and characteristics of this type of extreme event. For what concerns the X-rays, we have the possibility of investigating in more detail the properties of the variability on fast(er) timescales (see F08). A broad assessment of the reliability of our assumption can be made by considering the likelihood of a large amplitude variation on a timescale shorter than, e.g., $250 \mathrm{~s}$, the cutoff we applied to our sampling of $\tau_{2}$ and $\tau_{1 / 2}$. The analysis of the fractional rate variability for $\Delta t$ between 32 and $250 \mathrm{~s}$ shows that the probability for $\Delta F / F \geq 20 \%$ is only $\sim 6 \%$.

3. We would also like to point out that it would be desirable to use not simply the probability distribution for the $\tau$-values but the probability for a given change in rate $\Delta F / F$ for each given $\tau$. However, despite the size of the RXTE data set, it is not possible to have a good enough sampling for $P(\Delta F / F, \tau)$ to constitute a significant improvement over the uncertainty inherent in the assumption that all $\Delta F / F$ are equally probable for a given $\tau$.

The same analysis performed for the $9-15 \mathrm{keV}$ light curve yields $t_{\text {peak }}=30.0 \pm 0.7 \mathrm{ks}(1 \sigma), P\left(t_{\text {peak }}>30.25 \mathrm{ks}\right) \simeq 39 \%$, and an estimate of the lag of the TeV peak of $\tau=0.73 \pm 0.80 \mathrm{ks}$, i.e., no measurable lag.

\subsection{X-Ray versus TeV Flux Correlation}

Comparison of the variability amplitudes (as opposed to phases) offers different constraints. As clearly illustrated in Figures 2 and 3 , the source shows stronger variability in the $\gamma$-rays than in the $\mathrm{X}$-rays: in fact, in all panels the flux scale for the TeV data spans a range that is the square of that of the rate scale used for the $R X T E$
PCA data, and the light curves run in parallel. This is confirmed in Figure 6, which shows $\gamma$-ray flux as a function of X-ray count rate in different $\mathrm{X}$-ray energy bands. The $\mathrm{TeV}$ data are binned on approximately 28 minute runs. The $R X T E$ count rates correspond to the average over intervals overlapping with the TeV observations (as indicated by the shaded boxes in Fig. 3), and the error bars represent their variance (height of the shaded boxes).

We fit the log-log data with a linear relationship (i.e., $F_{\gamma} \propto F_{\mathrm{X}}^{\eta}$ ), which provides a satisfactory description in all cases. The best-fit slopes are reported in Table 4 (top row), along with their errors. We also analyzed the $\mathrm{X}$-ray/ TeV correlation for different sections of the campaign, and individually for each night, with the intent of looking for possible variations. Individual nights' plots are shown in Figure 7. In Table 4 we report the best-fit correlation slopes for the best single nights and for a few combinations of consecutive nights $(1+2,4+5,6+7$, and $5+6+7)$.

It is worth noting that again the March $18 / 19$ (day 1) X-ray/ $\gamma$-ray observations provide the best case study, for the large amplitude of variability, likely ensuring that the observed amplitude is close to the intrinsic one. The presence, and contribution, of a steady (variable on a longer timescale) emission diluting the flaring one could alter the perceived amplitude of flares. This is a long-standing issue that is difficult to address, but in this respect the March 18/19 flare is a unique event.

The unprecedented quality of this data set enables us not only to establish the existence of the correlation between the $\mathrm{TeV}$ and $\mathrm{X}$-ray luminosities, but also to start unveiling some of its more detailed characteristics, e.g., its evolution with time. The emerging picture is complex. There are several observational findings that we would like to point out.

1. The first, most direct and general observation is that the TeV flux shows a definitive correlation with the X-ray rate, for all X-ray energy bands (see Fig. 6). If we consider the entire week-long data set, 105 data pairs, the correlation is approximately linear (see Table 4). The same is apparent when looking at the data binned over a 1 day timescale, as shown in Figure $8 a$. 

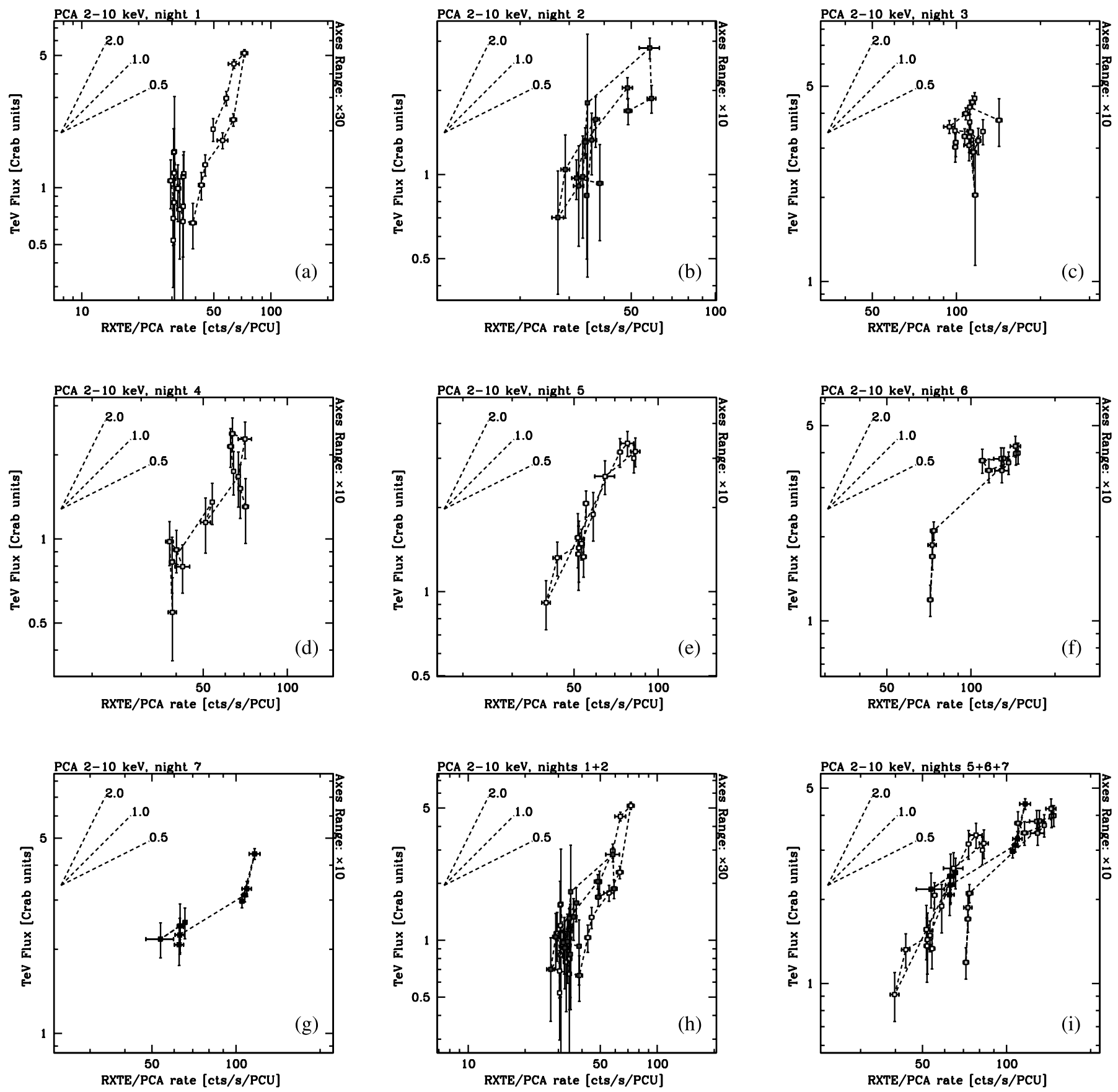

Fig. 7.- Plot of the Whipple + HEGRA TeV flux vs. X-ray $2-10 \mathrm{keV}$ count rate for each individual observation night, and the combination of nights $1+2$ and $5+6+7$. The axis range is 10 times in all panels except for those involving the March 19 (night 1) data, whose variation range is larger ( 30 times). Some of the symbols are shaded in gray to help recognize different sets.

2. A more careful inspection of the flux-flux diagrams, however, suggests a richer phenomenology. In fact, we may be observing a series of parallel flux-flux paths, individually obeying a steep (e.g., quadratic) trend but that taken together produce a rather flat envelope producing the linear trend emerging for the global cases, because of a drift of their barycenters. There is indeed a secular increase of the source brightness over the course of the campaign, and it seems to be more enhanced in the X-ray. Its amplitude is of the order of the intranight brightness variations, thus altering the X-ray $/ \gamma$-ray correlation on longer timescales. Figure $8 b$ shows how the regions covered by nightly data shift from day to day, while broadly maintaining an ap- proximately quadratic intranight flux correlation trend in most cases.

There is thus an intriguing hint that there might be a split between the correlation observed on short (hours) timescales and that apparent on longer (days) timescales, once faster variations are smoothed out.

3. There may be two different (luminosity related) regimes for the X-ray/TeV flux correlation. By splitting the data into two sections of significantly different average brightness level, days $1+2$ (with or without the preflare noisy HEGRA data section) and days $6+7$ (or $3+6+7)$, we can see that the source seems to exhibit two different behaviors: the TeV versus X-ray 

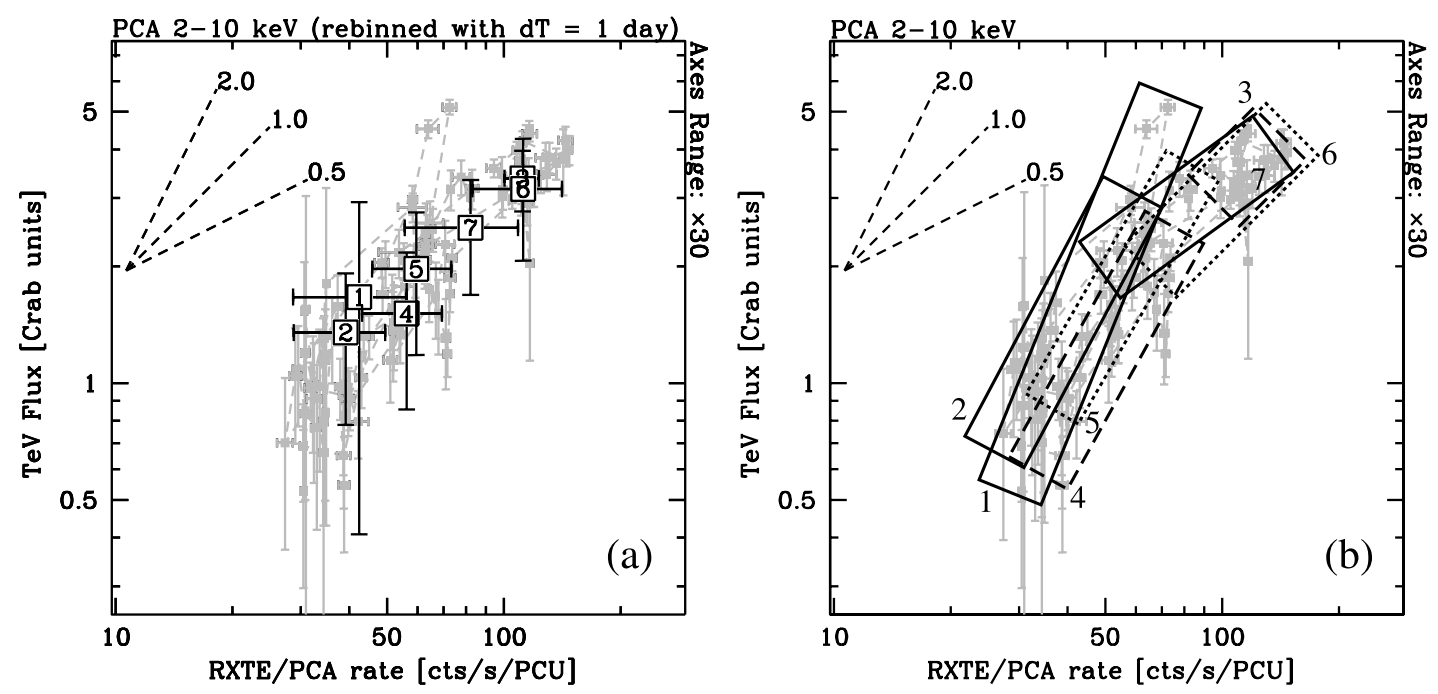

FIG. 8.- (a) X-ray vs. $\gamma$-ray 1 day averaged brightnesses. The error bars represent the variance during the interval, which can be considerable. The correlation is approximately linear (see Table 4). Numbers refer to the campaign night sequence. (b) Boxes (approximately) representing the regions of the diagram occupied by the data of each individual night. The combination of steep(er) intranight and flat(ter) longer term (due to shift of the barycenters) correlations is more easily shown. Both plots are on the same axis scale and range. Box contours are solid for nights 1,2, and 7; long-dashed for 3 and 4; and dotted for 5 and 6.

relationship is significantly steeper for the day $1+2$ subset, with values of $\eta$ for all four PCA energy bands larger than $\eta=$ $1.82( \pm 0.12)$, versus all values smaller than $\eta=1.03( \pm 0.14)$ for days $6+7$ (Table 4 ).

4. For two nights ( 1 and 5 ) the flux-flux diagram is very tight, with all points lying on a very narrow path. In these cases the $\mathrm{TeV}$ flux increases more than linearly with respect to the X-ray rate. For the flare of March 19 the correlation is superquadratic at all energies (Table 4). Moreover, for these two nights the light curves encompass a full flaring cycle; i.e., we can follow the complete evolution of an outburst, rising and decaying. In both cases the paths of the rising and decaying phases in the flux-flux diagram overlap perfectly.

5. There is no significant change of the slope of the correlation with the choice of X-ray energy band, except for the case of the full week-long data set. A flatter correlation slope for harder X-rays would be expected because of the intrinsically higher amplitude of the variability of the synchrotron component toward higher energies (if we are already above the peak energy; e.g., Fossati et al. 2000a). In fact, the relative variance, $\sigma_{F} /\langle F\rangle$, increases with energy, changing from $\simeq 0.45$ to $0.48,0.52$, and 0.56 for $2-4,4-6,6-8$, and $9-15 \mathrm{keV}$, respectively. This change fully accounts for the flattening of the X-ray $/ \gamma$-ray correlation slope. The effect is not observed for smaller subsets of data probably because of the lower statistics.

The departure of the $20-60 \mathrm{keV}$ band from this trend could instead be justified by considering that the flux in this band may comprise a contribution from the onset of the inverse Compton scattering, which could be regarded as constant because it would be varying on much longer timescales. However, this hypothesis does not seem to be supported by the data, because although they are very noisy and have limited energy leverage, the HEXTE data are consistent with the extrapolation of the steep PCA power law. Alternatively it is possible that the difficult background subtraction of the low-count-rate HEXTE data reduces the intrinsic dynamic range of the X-ray variations, thus steepening the correlation.

These observational findings have important implications for the physical conditions and processes responsible for the variability in the scattering region, as discussed in $\S 4$.

\subsubsection{Comments}

Before we proceed to discuss the observational findings, we would like to put forward a few additional comments concerning some aspects of the derivation and interpretation of the flux-flux correlation.

1. For simplicity we performed the brightnesses correlation analysis using count rates for the $R X T E$ data. A proper conversion to flux units requires fitting a model to the data for each short subinterval, and it would introduce a different source of uncertainty. We tested the correspondence between count rates in RXTE PCA bands and model fluxes for a broken power-law model with different spectral indices and break energy positions covering the range of values observed in 2001 March (for full account of the spectral analysis please refer to F08). For the $2-10 \mathrm{keV}$ band, the correlation between count rate and flux is slightly tilted, in the sense of slightly less than linear increase of the flux with rate, flux $\sim$ rate $^{0.9}$. This would thus further steepen the $\mathrm{TeV} / \mathrm{X}$-ray flux-flux correlation if computed with the X-ray flux. The effect is small and it is not present when narrower energy bands are considered.

2. Rebinning the data alters the variance of the light curves, and if the effect is different for X-rays and $\gamma$-rays (namely, if their intrinsic power spectra are different), it could potentially bias the slope of the correlation. The comparison of the change of variance of X-ray and $\gamma$-ray (starting from the $256 \mathrm{~s}$ binned one when possible) light curves for different rebinnings suggests that the effect is at most of the order of $10 \%$. The effect is small in comparison with the overall range spanned by the data, which is of the order of a factor of at least 5 for the week-long data set. Hence, we deem the effect of the choice of time binning on the determination of the flux-flux correlation slope not significant.

3. Since we are measuring the fluxes in limited energy bands, the slope of the relation also depends on the position of the synchrotron and $\gamma$-ray peaks with respect to the observed energy bands. The reason is that as the peak moves from lower frequency into the bandpass of a detector, a small change in the peak position yields a larger variation of the flux. A simple shift in frequency would be degenerate with a true increase in luminosity. Once the 
spectral peak falls within the bandpass and is shifting within it, this spurious effect becomes unimportant. In a very simplified case, taking Mrk 501 as test SED, Tavecchio et al. (2001) showed that the $\gamma$-ray versus X-ray flux relationship predicted for variability simply due to a change in the maximum particle energy (and in turn synchrotron peak energy) can vary between flatter than linear to steeper than quadratic (however, the effect was enhanced by the fact that the authors compared monochromatic fluxes). Katarzyński et al. (2005) performed a thorough analysis of the effect of the position of observed energy bands with respect to the synchrotron or IC peak energies in the context of the X-ray versus $\gamma$-ray brightness correlation and found that it can change the slope over a broad range of values, including linear and quadratic. But this apparent freedom is lost if data following the full evolution of a flare are available. In fact their conclusion with respect to an outburst developing like that of March 19 is that explaining the observed correlation by means of specific choices of spectral bands is problematic and would require very contrived assumptions.

The characteristics of the X-ray variability itself seem to evolve during the campaign. In particular, it is important to recall that the spectrum becomes significantly harder over the course of the week-long campaign, accompanying a gradual brightness increase. Rather than a caveat this is probably a point in support of the apparent change of X-ray $/ \gamma$-ray behavior between the first and second part of the week. The spectral analysis of the 2001 March Whipple data, reported separately by Krennrich et al. (2003), showed that the $\mathrm{TeV}$ spectra also significantly hardened between March 19 and 25. The spectral indices for a power-law fit with exponential cutoff (fixed at $4.3 \mathrm{TeV}$ ) shift from $\Gamma \simeq 2.3$ to $\simeq 1.8$ $( \pm 0.15)$, i.e., suggesting that the IC peak moved from below to within the Whipple bandpass (i.e., in the latter case the $\gamma$-ray emission would peak at about $1 \mathrm{TeV}$ ). RXTE spectra present a similar picture of the X-ray evolution, namely, that the synchrotron peak shifted into the PCA bandpass. Broken power-law fits show that the lower energy spectral index becomes harder than $\Gamma=2$ (F08). Unfortunately even with the available statistics, because of the limited energy leverage, it is not possible to pinpoint robustly the energy of the synchrotron peak and its evolution (as was the case with BeppoSAX).

If the peak of one component (synchrotron or IC) moves into the observed band, we would then be observing the variations of a lower, possibly below peak, section of the electron spectrum, instead of the more highly variable higher energy end. Depending on whether this happens to both peaks or just one, we expect to observe a different phenomenology: for example, if this happens only for the TeV band the correlation with the X-ray data should become flatter (smaller $\gamma$-ray variation for a given $\mathrm{X}$-ray one). This might explain the apparent change of the fluxflux correlation trend between the beginning and end of the weeklong campaign.

However, it is worth noting that Krennrich et al. (2003) find that during the flare of March 25, a flux variation larger than a factor of 2 does not seem to be accompanied by any spectral change. Given the characteristics of the spectra, namely, the fact that the IC peak at most moved marginally within the observed band, this achromaticity cannot be convincingly ascribed to the fact that Whipple was observing the lower energy shoulder of the IC peak. The possibility that it is intrinsic has to be contemplated.

Therefore, the possible change of the X-ray/ TeV flux correlation may also be attributed to some intrinsic effect, possibly related to the longer term increase of luminosity.

\subsection{X-Ray versus TeV Spectra and Spectral Energy Distributions}

\subsubsection{Intranight $X-R a y / \gamma$-Ray Spectra Pairs}

Besides the unprecedented quality of the X-ray and $\gamma$-ray light curves that we have illustrated and discussed in the preceding sections, the 2001 March data set affords us a unique opportunity to follow the spectral evolution itself, with a time resolution that allows meaningful intraflare analysis. Detailed SED-snapshot and time-dependent modeling analyses are beyond the scope of this paper and will be presented in a forthcoming publication. Here we present the subset of X-ray/ $\gamma$-ray spectra for the March 19 event (Whipple) and for the March 21/22 and 22/23 flares (HEGRA, presented by Aharonian et al. 2002).

A summary gallery of the pairings of X-ray and $\gamma$-ray spectra for these flares is shown in Figure 9. For reference we also plotted some historical observations.

It is worth noting that this gallery does not include the highest luminosity X-ray states, nor in general (i.e., irrespective of simultaneous $\gamma$-ray data), neither among the intervals matching $\mathrm{TeV}$ observations. On the other hand, the peak of the March 19 flare does constitute the most luminous TeV spectrum of the 2001 campaign, and in fact it matches the spectrum and luminosity of the most intense flare ever recorded for Mrk 421, that of 1996 May 7 (Zweerink et al. 1997).

For ease of comparison we prefer to adopt the same axis scales for X-ray and $\gamma$-ray, which makes the variability of the $R X T E$ spectra not as easily noticeable as that of Whipple/HEGRA spectra. Nevertheless, the level of variability can be appreciated by comparison with the reference historical spectra.

We would like to highlight a few observational findings. The peaks of the synchrotron and IC components never cross into the telescopes bandpasses, despite the relatively large luminosity variations. Increases in brightness are accompanied by significant spectral hardening, but there is no compelling sign that this is also accompanied or caused by a shift of the SED peak energies. Among the data presented here, the only instances when the synchrotron peak might be or is directly detected are the spectra for the March 22/23 flare. It indeed seems that the high-energy tails move between hard and soft states as if pivoting with respect to unobserved lower energy parts of the spectrum, possibly the synchrotron or IC peaks. This is suggested by the observation that in most cases the lowest energy data points in successive spectra are approximately at the same level, whereas we would expect some upward shift in both the case of variations due to a change of energy of the SED peak and the case of an overall increase of luminosity around the SED peak.

\subsubsection{Spectral Energy Distributions}

In Figure 10 we show selected simultaneous X-ray and $\gamma$-ray spectra for the 2001 March campaign, together with a collection of historical multiwavelength data (see Fig. 10 for details).

In particular Figure $10 a$ shows the data for the peak of the March 19 outburst and Figure $10 b$ the preflare interval for March 22/23. These two are quite representative of a bright and hard case and a fainter and soft case. We tried to model these sparse SEDs with a single-zone homogeneous SSC model (e.g., Ghisellini et al. 1998), and example fits are plotted along with the data.

Although the simultaneous data coverage is limited to optical flux and the X-ray and $\gamma$-ray spectra, a coarse search of the parameter space for a good SSC model fit showed that the constraints are nonetheless very strong. This is true even though we made no attempt at taking into account self-consistently the abundant 

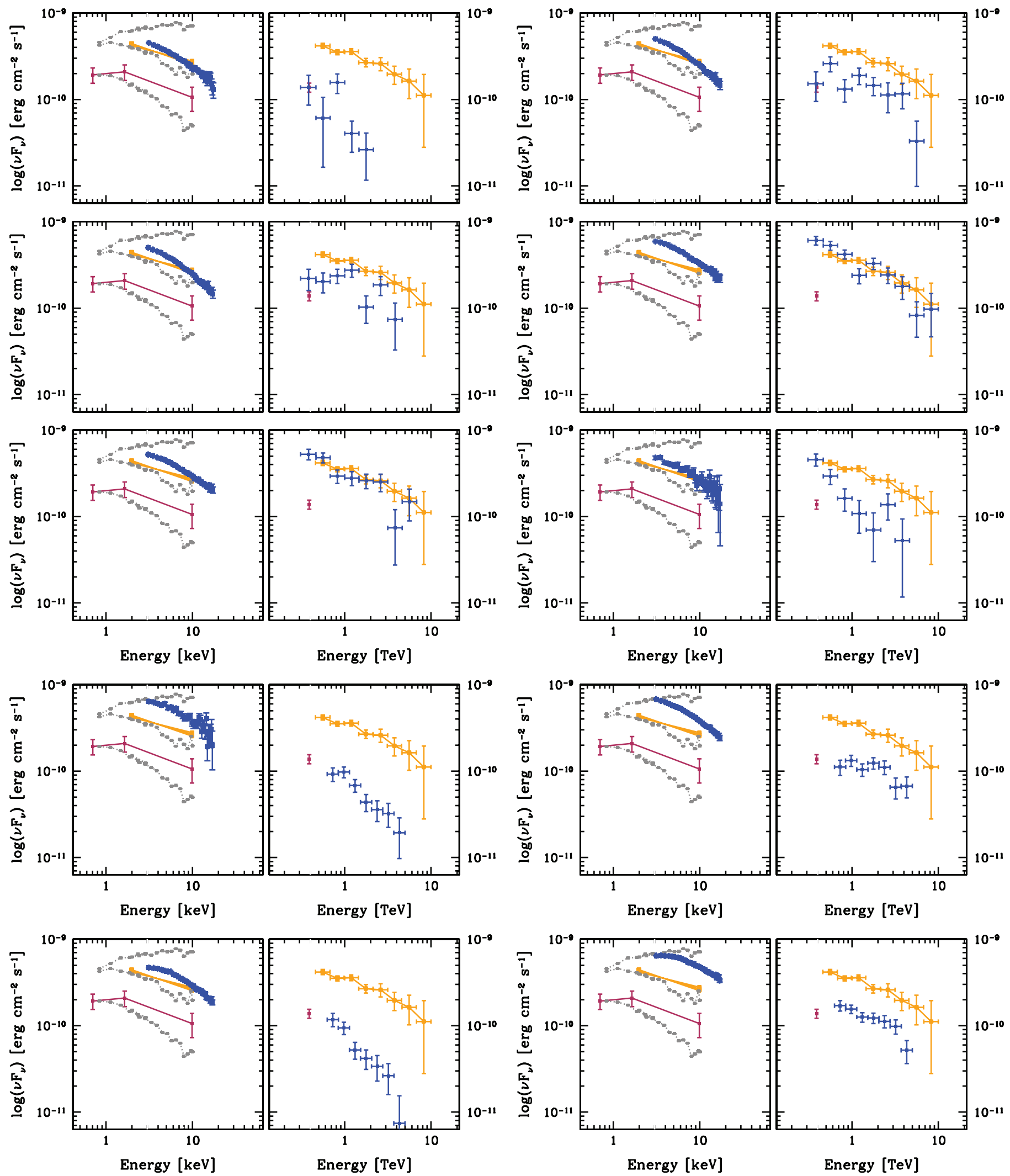

FIG. 9.-Gallery of RXTE and Whipple/HEGRA spectra pairs (blue symbols). Time elapses left to right, top to bottom. The top six panels refer to March 19 (Whipple); approximate times are (UTC) 05:39, 07:04, 07:34, 08:33 (flare top), 08:59, and 09:30. The bottom two pairs are for March 21/22 and 22/23, with HEGRA data (Aharonian et al. 2002; preflare and flare). For reference we also plot (1) the simultaneous observations of the 1994 May flare reported by Macomb et al. (1995; maroon symbols, threepoint X-ray spectrum and single flux point at $0.4 \mathrm{TeV}$, at $\approx 10^{-10}$ ), (2) the highest state observed in 1996 (Zweerink et al. 1997; orange symbols, X-ray power law from ASCA and $0.4-10 \mathrm{TeV}$ spectrum by Whipple), and (3) the 0.7-10 keV X-ray spectra (denser light gray points) for the lowest and highest state (bottom to top) during the BeppoSAX 1998 campaign (Fossati et al. 2000b) and the highest BeppoSAX 2000 state (F08). 

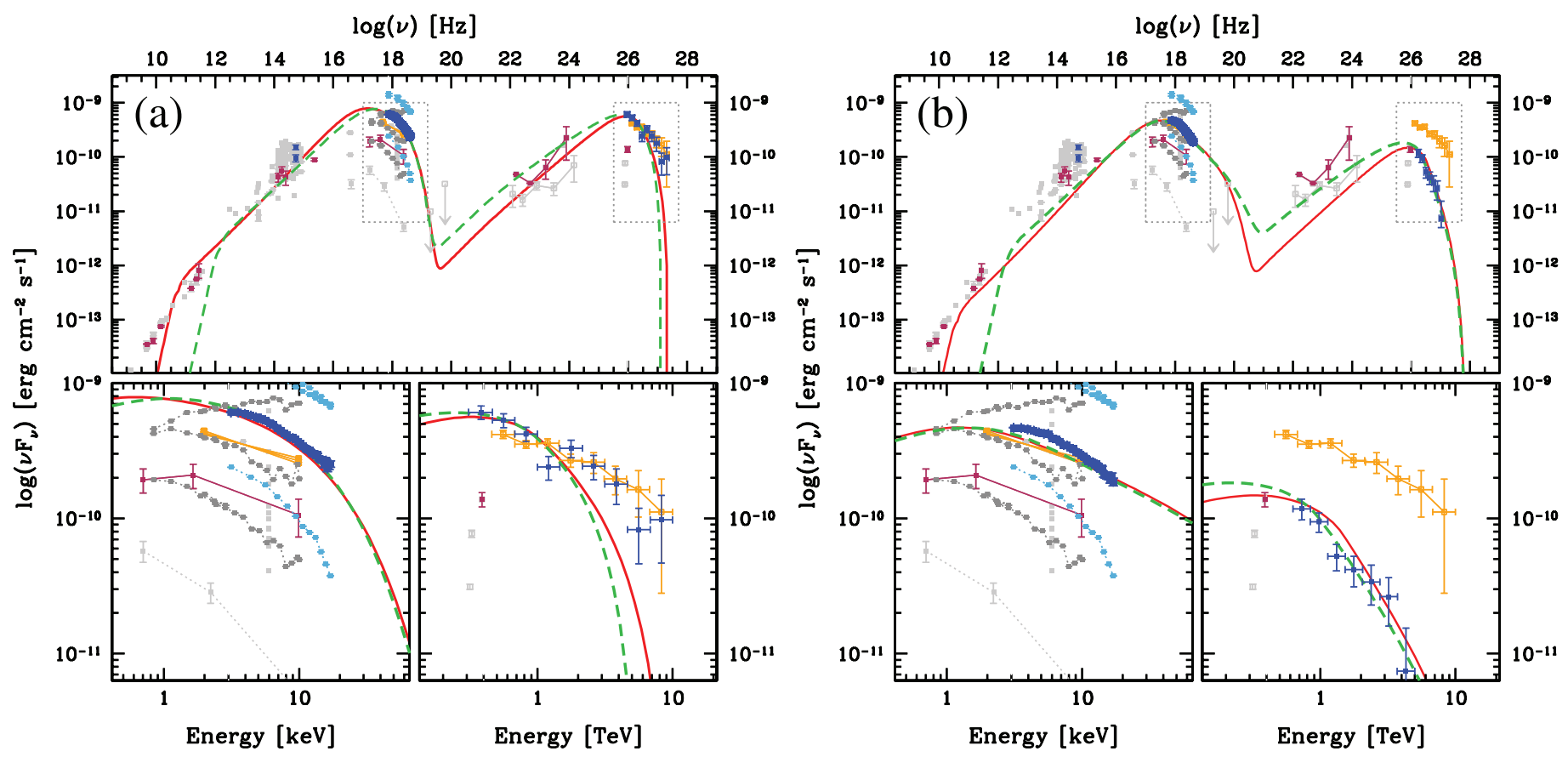

FIG. 10.- Spectral energy distributions for two epochs during the 2001 March campaign. (a) Time around the peak of the March 19 flare. (b) Low ( preflare) state observed by HEGRA on March 22/23 (HEGRA spectra from Aharonian et al. 2002). Simultaneous 2001 data are shown in blue. For the optical we plot the highest and lowest fluxes observed during the campaign. The sparser, connected, light blue, 3-20 keV X-ray spectra show the highest and lowest X-ray observed states. For reference, we plot in gray multiwavelength data from a collection of historical data from NED and Macomb et al. (1995). The maroon points are the simultaneous observations for 1994 May (Macomb et al. 1995). For the description of some of the X-ray and $\gamma$-ray data see also Fig. 9. The solid red lines represent fits with a simple one-zone homogeneous SSC model with $B \simeq 0.1-0.15 \mathrm{G}, \delta=20-25$, and $R_{\mathrm{blob}}=10^{16} \mathrm{~cm}$. The dashed green SED models are for extreme cases, with $B \simeq 1 \mathrm{G}, \delta=100$, and $R_{\text {blob }}=(0.5-1) \times 10^{14} \mathrm{~cm}$.

information available along the time axis, such as the time-resolved spectral variability. One general difficulty encountered while fitting the SSC model is that the TeV spectra are typically harder than what can be predicted. As we illustrate in $\S 3.5 .4$, this is in part due to the effect of the Klein-Nishina (K-N) decrease of the scattering efficiency, canceling the contribution from the selfCompton of the electrons and photons emitting or emitted above the synchrotron peak.

\subsubsection{B- $\delta$ Diagnostic Plane}

Since we can estimate the energies and luminosities of the synchrotron and IC peaks with reasonable accuracy, in the context of a single-zone SSC model we can draw the locus allowed by a given SED in the $B-\delta$ parameter space (e.g., Tavecchio et al. 1998). Besides this primary piece of information, measurements or estimates of several other quantities (and their combinations) can be exploited to set additional constraints on the $B-\delta$ relationship. These include, for instance, peak luminosities, cooling times, variability timescales (or source size), and intraband time lags.

An example is shown in Figure 11, for March 22/23. The gray band represents the constraints set by our estimate of the peak positions. The peak luminosities yield a few additional lines in the $B-\delta$ plane, in particular the particle-magnetic field equipartition. A requirement on the cooling time of the peak-emitting particles, for instance to be shorter than the typical variability timescale (e.g., $10 \mathrm{ks}$ ), translates into an excluded wedge in the lower right part of the plane. The two different lines, meeting at the equipartition line, correspond to synchrotron- or IC-dominated cooling regimes.

Detection or an upper limit on the value of intraband X-ray lags also sets a lower boundary to the allowed region in the diagram. In Figure 11 we draw the limit for a hypothetical $2 \mathrm{ks}$ lag within the PCA bandpass. Shorter lags, or upper limits on them, move this line upward. It is worth noting that the detailed cross-correlation analysis of the X-ray data set (F08) does not yield any reliable intraband lag detection. In particular, no lags have been found in the analysis of all short, single-orbit subsets with significant variability features (a few dozen), and in most cases the upper limit is of the order of a few hundred seconds, $\simeq 200-300$ s (the corresponding line in Fig. 11 would be about 0.5 decades, 3 times, higher).

Finally, we can draw in the $B-\delta$ plane the dividing line between the Thomson and K-N scattering regimes, for the SED peaks.

One of the largest sources of uncertainty for the determination of the allowed region in the $B-\delta$ plane is the position of the IC peak, because

$$
\frac{B}{\delta} \propto \frac{\nu_{\text {peak, sync }}}{\nu_{\text {peak }, \text { IC }}^{2}}
$$

for scattering in the K-N regime. This also means that any consideration based on this diagnostic plane is subject to the uncertainty about the details of the $\mathrm{TeV}$ photon absorption by the diffuse infrared background. The models shown here include the effect of the IR absorption, following the low-intensity model prescription of Stecker \& De Jager (1998). For our limited modeling purpose the exact choice of IR background absorption model is not critical.

Figure 11 shows the loci and limits obtained for a SED similar to that in the right panels of Figure 10 (March 22/23), for which we obtained a satisfactory SSC model fit. The relevant observational parameters are reported in the plot. All constraints are satisfied by a model having a magnetic field of $B \simeq 0.15 \mathrm{G}$, a Doppler factor $\delta \simeq 20$, and a blob size of $R \simeq 10^{16} \mathrm{~cm}$, i.e., within the range regarded as standard in SSC modeling (see Fig. 11, circle, and Fig. 10b, solid line). 


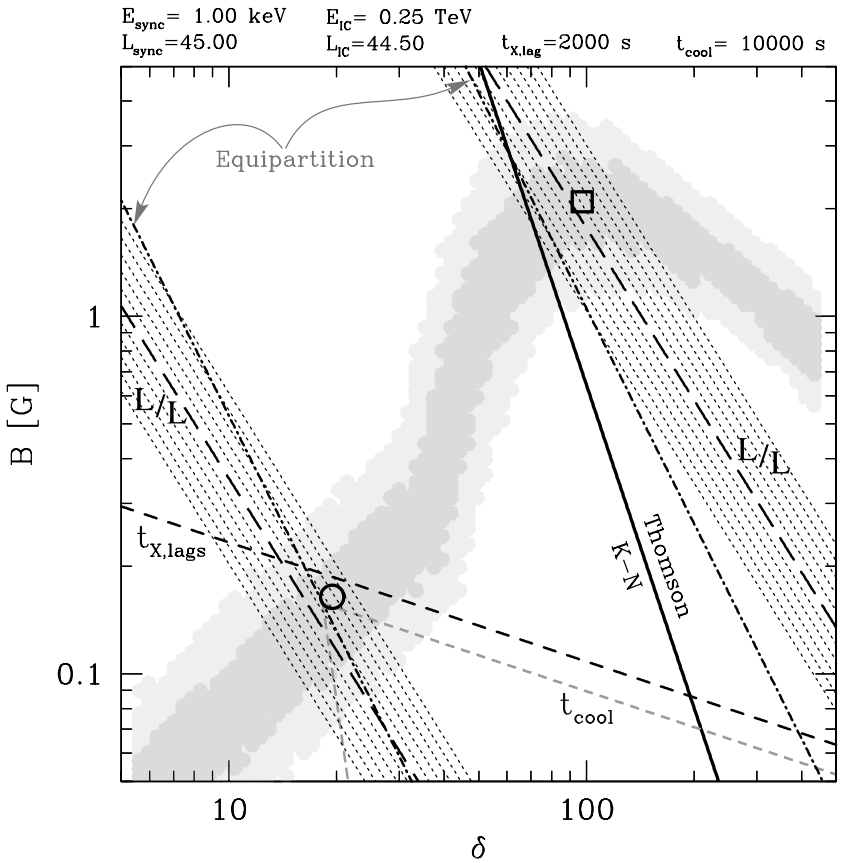

FIG. 11. $-B-\delta$ plane for a set of parameters $\left(\nu_{\text {peak, } \mathrm{X}}, \nu_{\text {peak, } \gamma}, L_{\text {peak, } \mathrm{X}}\right.$, and $L_{\text {peak, } \gamma}$ ) representative of the 2001 campaign. The gray locus crossing the plane shows the constraint set by the synchrotron and IC components' peak frequencies (adopted values are shown above the figure). The long-dashed steep lines show the range allowed by the inferred peak luminosities. The dot-dashed steep lines mark the equipartition between $U_{B}$ and $U_{\text {rad }}$. There are two sets of long-dashed and dot-dashed lines: the left ones correspond to a standard case of $R=10^{16} \mathrm{~cm}$, while those on the right refer to the case of $R=5 \times 10^{13} \mathrm{~cm}$. The dotted regions are meant to represent the effect of a factor of 3 uncertainty on the main parameters. The thick black line is the approximate analytical boundary between Thomson and K-N scattering regimes. The gray dashed lines mark the combination of parameters yielding a $10 \mathrm{ks}$ cooling time for electrons emitting the synchrotron peak. The bottom right corner wedge delimited by them is inconsistent with this imposed (putative) limit. The short-dashed black line is the lower bound allowed by a hypothetical X-ray intraband lag of $2 \mathrm{ks}$. The open circle marks the parameter choice for the SED model shown by the solid red line in Fig. 10b. The open square instead marks a possible choice of parameters in the Thomson regime region of the $B-\delta$ plane, the dashed green SED in Fig. $10 b$.

This analysis shows that for this choice of parameters the scattering producing the $\mathrm{TeV}$ emission occurs in the $\mathrm{K}-\mathrm{N}$ regime. It is in principle possible to shift the sweet spot in the upper corner of the diagram into the Thomson regime region by adopting a much smaller source size $\left(R \simeq 5 \times 10^{13} \mathrm{~cm}\right)$ and in turn $B \simeq 2 \mathrm{G}$ and $\delta \simeq 100$ (Fig. 11, square), and indeed a similarly satisfactory SSC fit to the snapshot SED can be obtained (Fig. 10b, dashed line).

A similar analysis was performed for the data of the March 19 flare peak, shown in Figure $10 a$, and also in this case the SED could be fit with both "standard" $\left(B=0.1 \mathrm{G}, \delta \simeq 20, R=10^{16} \mathrm{~cm}\right)$ and "extreme" $\left(B=1.0 \mathrm{G}, \delta=100, R=10^{14} \mathrm{~cm}\right)$ parameters (corresponding SEDs are shown as solid and dashed lines, respectively, in Fig. 10).

Other considerations can help to discriminate between these scenarios, for instance, arguments concerning time variability properties or the viability of having such an extreme Doppler factor and blob size.

\subsubsection{TeV Spectral Decomposition Analysis}

In order to try to understand the observed correlated variability between X-ray and $\mathrm{TeV}$ fluxes, it is interesting to take a deeper look at the composition of the emission in the TeV band, in terms of which electrons and seed photons contribute to the flux at different energies. This analysis is somewhat model dependent,

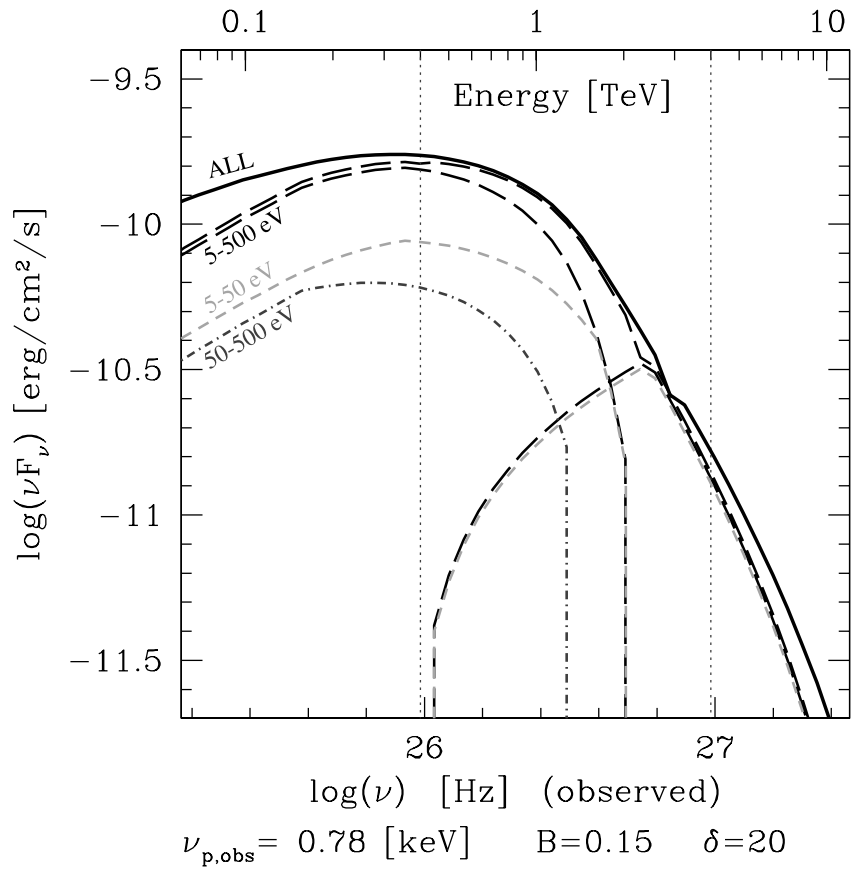

FIG. 12.- Modeling of the inverse Compton peak for the SSC model shown as a solid red line in Fig. 10b. The IC emission is split into different components a la Tavecchio et al. (1998). The long-dashed black lines show the emission obtained by restricting the synchrotron seed photons to the $5-500 \mathrm{eV}$ range. It is split in two by considering separately electrons emitting in synchrotron below and above $2 \mathrm{keV}$ : the lower energy section is thus coming from $5-500 \mathrm{eV}$ photons scattered by electrons up to $2 \mathrm{keV}$. This latter is then further split into the contributions from $5-50 \mathrm{eV}$ (light gray short-dashed line) and 50-500 eV photons (dark gray dotdashed line). The thick black solid line is the full IC component, i.e., including all seed photon contributions. The vertical dotted lines mark the $0.4-4 \mathrm{TeV}$ band.

and we are only showing it for the "standard" parameter choice introduced above.

The idea is similar to the treatment discussed by Tavecchio et al. (1998), where they split the IC component into four components, produced by the combinations of electron and synchrotron photons below (L) and above $(\mathrm{H})$ the synchrotron peak (for electrons the split is done at the energy mapped to the latter). For TeV blazars the conditions are such that the component $(\mathrm{H}, \mathrm{H})(\mathrm{elec}-$ trons and photons both above the peak) is strongly depressed and becomes negligible. The same holds true for the ( $\mathrm{L}, \mathrm{H})$ component (Tavecchio et al. 1998).

The same approach can be extended to an arbitrary split of the primary components, aiming at identifying more precisely the origin of the electrons and photons.

In Figure 12 we show the IC peak for the same model on which we have focused above. We adopt as the breaking point for the electron spectrum the energy corresponding to an observed synchrotron emission at $2 \mathrm{keV}$, because this is just below the $R X T E$ PCA bandpass and this splitting allows us to divide the electrons between those whose synchrotron we observe and those we do not. We consider all electrons but restrict the allowed seed photons to those in the range 5-500 eV, observer's frame. Different line types correspond to different ranges of seed photons within this band. The long-dashed black lines show the full emission by $5-500 \mathrm{eV}$ photons and electrons below and above $2 \mathrm{keV}$, with its decomposition in the two contributions. It is clear that most of the emission at around $1 \mathrm{TeV}$ is accounted for by these photons scattered by sub-keV electrons. The gray short-dashed and dot-dashed lines show a further split of the photons in the 5-50 and 50$500 \mathrm{eV}$ bands, with the former contributing about $60 \%$ of the total at around $1 \mathrm{TeV}$. 
Because of the K-N effect, there is no emission by self-Compton by electrons observed in synchrotron in the X-ray band. Moreover, the (non-self) IC contribution by X-ray-observed electrons is weak and at high enough energy that we can regard it as irrelevant to the effect of the observed $\mathrm{TeV}$ rate variability. The latter is dominated by a lower energy section of the bandpass, around $\simeq 1 \mathrm{keV}$.

The K-N depression of the scattering of the higher energy photons and electrons cuts off the $(\mathrm{H}, \mathrm{H})$ and $(\mathrm{H}, \mathrm{L})$ contributions and makes the TeV spectrum always steeper than the X-ray one, at least in the more minimalist scenarios. Indeed in first approximation the power law of the high-energy tail of the IC component tends to a value $\alpha_{\mathrm{K}-\mathrm{N}} \simeq 2 \alpha_{2}-\alpha_{1}$, where $\alpha_{1}$ and $\alpha_{2}$ are the spectral indices below and above the synchrotron peak (Tavecchio et al. 1998). This is always steeper than $\alpha_{2}$, and for fiducial values of $\alpha_{1}$ and $\alpha_{2}$ it is so by $\Delta \alpha \gtrsim 0.5$.

In the more extreme scenario contrived to shift the scattering into the Thomson regime, the IC peak composition is indeed different. In particular, as expected, there is a more even contribution by three of the four components. The $(\mathrm{H}, \mathrm{H})$ component is still negligible. Photons up to $2-3 \mathrm{keV}$ are effectively scattered, and there is a self-Compton contribution to the emission at $\simeq 1 \mathrm{keV}$. Therefore, a much more direct connection between the two observed bands is afforded by the more exotic scenario, and in turn the observed correlated variability would be more readily explained.

\section{CONCLUSIONS}

The correlation between the variations in the X-ray and TeV bands is confirmed with unprecedented detail, supporting the idea that the same electron distribution, in the same physical region, is responsible for the emission in both energy bands. However, the details of these findings pose a serious challenge to the emission models. Here we would like to sketch a few selected outstanding issues raised by the correlated variability. We refer to a forthcoming paper for an in-depth analysis comprising more extensive modeling (F08).

1. If modeled within the realm of standard values for magnetic field, Doppler factor, and source size, the IC scattering responsible for the observed $\mathrm{TeV}$ emission occurs in the $\mathrm{K}-\mathrm{N}$ regime. This means that with X-ray and $\gamma$-ray observations, although we seem to be observing regions of the spectrum that are very similar to each other for what concerns their position with respect to the SED peaks, we are not tracking the evolution of the same electrons (and photons). The extent of the phase and amplitude correlation of the X-ray and $\gamma$-ray variations is, however, remarkable, and this sets broad constraints on the characteristics of the processes responsible for and governing the variability (e.g., acceleration/ injection of particles, dominant cooling mechanism).

2 . In this context, the observation in the K-N regime of a quadratic relationship between synchrotron and IC variations (which would be naturally produced in the Thomson regime because of the effectiveness of self-Compton) constrains the electron spectrum variations to occur over an energy band broad enough to affect also the IC seed photons. Moreover, this variation must be essentially achromatic (i.e., just a change in normalization), otherwise the extra energy-dependent factor would produce an observable effect.

3 . The observation that the flux-flux path of the better observed flare decay closely follows the bursting path introduces a further complication. If the flare decay is governed by the cooling of the emitting electrons we do not expect the quadratic relationship to hold during the decaying phase. In fact, given the energy-dependent nature of synchrotron (and IC) cooling, with $\tau_{\text {cool }} \sim E_{\mathrm{ph}}^{-1 / 2}$, the $50 \mathrm{eV}$ seed photons cool on a longer timescale, e.g., $\sim 10$ times longer than the timescale for photons observed at $\gtrsim 3 \mathrm{keV}$. The possibility that the electrons contributing to the bulk of the TeV emission also have lower energy than those observed in the X-ray compounds the problem. This means that during the flare decay the X-ray and $\gamma$-ray brightnesses should follow something like a linear relationship, because the IC ( TeV) emission will just reflect the evolution of the electron spectrum, scattering a steady seed photon field.

Plain radiative cooling does not seem to match these observations. A viable mechanism explaining the flare evolution should allow the concurrent cooling of a broad portion of the electron distribution. On the other hand, brightness variations are accompanied by large spectral changes, and in most cases they are very suggestive of acceleration - or injection - of the higher energy end of the electron population.

4. Another recurring discrepancy between data and simple one-zone SSC modeling is that of the TeV spectral shape, which is often harder than model predictions. As we illustrated in the previous section the K-N effect plays an important role in this respect. A more careful analysis is warranted, but it is worth noting that one alternative option for addressing this problem is that of considering the effect of additional IC components, off photons external to the blob. Błażejowski et al. (2005) showed that a multicomponent model seems to be required to fit the 2003-2004 observations, and it might mitigate the discrepancy in the TeV spectrum. Ghisellini et al. (2005) discuss the effect of including the effect of the radiation emitted by the putative lower Lorentz factor outer layer of the jet, namely, as source of additional seed photons for IC.

5. Exotic scenarios could address some of these issues, namely, by allowing the IC scattering to occur in the Thomson regime and hence the self-Compton to be effective, thus reinstating the close relationship between the photons and electrons tracked by X-ray and $\gamma$-ray observations. One such scenario that we discussed briefly would call for very high values of the Doppler beaming factor, which would reduce the intrinsic energies at play, higher magnetic field, and very small size of the emission region. In fact recently there has been some interest in less conventional modeling of TeV blazars (e.g., Krawczynski et al. 2001; Rebillot et al. 2006 for Mrk 421). However, high Doppler factor scenarios raise a series of new issues, or reopen some that have been settled for the more traditional model. First of all, the fact that the beaming cone of the radiation emitted by such a fast blob is going to be much narrower has to be reconciled with the population statistics of blazars and radio galaxies, their putative parent population (Urry \& Padovani 1995). One way of doing it would be to imagine that the jet comprises a very large number of small high Lorentz factor blobs, fanning out filling a wider cone, with an aperture consistent with the unification statistics. This would constitute a quite radical change in the jet structure from the current one where emission is thought to come from internal shocks. There are also implications concerning the statistical properties of the variability, which would likely be due to the combination of the bursting of different blobs, most likely uncorrelated.

The richness and depth of the X-ray and $\gamma$-ray data of the 2001 March campaign presented in this paper raise the bar for models. The aggregate characteristics illustrated here already challenge the simple traditional SSC model and the SED-snapshot approach. In order to answer the questions raised by these observations it is of paramount importance to exploit fully the time-axis dimension into the modeling and take a dynamical approach.

The data time density and brightness (and so statistics) are unparalleled, enabling time-resolved spectroscopy on timescales of the order of the physically relevant ones, hence allowing us to 
model the phenomenology self-consistently and minimizing the need (freedom) to make assumptions as to how to connect spectra taken at different times.

It is likely that this data set is going constitute the best benchmark for time-dependent modeling for some time, despite the great progress made by ground-based $\mathrm{TeV}$ atmospheric Cerenkov telescopes in the last few years, because of the difficulty of securing long uninterrupted observations with Chandra and XMM-Newton.

G. F. thanks Jean Swank, Evan Smith, and the RXTE GOF for their outstanding job, in particular for implementing the best
RXTE scheduling possible. G. F. has been supported by NASA grants NAG5-11796 and NAG5-11853.

The Whipple Collaboration is supported by the US Department of Energy, the National Science Foundation, the Smithsonian Institution, PPARC (UK), NSERC (Canada), and Enterprise-Ireland.

This research has made use of NASA's Astrophysics Data System and of the NASA/IPAC Extragalactic Database (NED), which is operated by the Jet Propulsion Laboratory, California Institute of Technology, under contract with the National Aeronautics and Space Administration.

Facilities: FLWO:10m, RXTE (PCA), HEGRA, FLWO:1.2m
Aharonian, F., et al. 2002, A\&A, 393, 89

Błażejowski, M., et al. 2005, ApJ, 630, 130

Blumenthal, G. R., \& Gould, R. J. 1970, Rev. Mod. Phys., 42, 237

Böttcher, M., \& Reimer, A. 2004, ApJ, 609, 576

Buckley, J. H., et al. 1996, ApJ, 472, L9

Coppi, P. S., \& Blandford, R. D. 1990, MNRAS, 245, 453

de la Calle Pérez, I., et al. 2003, ApJ, 599, 909

Dermer, C. D., Schlickeiser, R., \& Mastichiadis, A. 1992, A\&A, 256, L27

Edelson, R. A., \& Krolik, J. H. 1988, ApJ, 333, 646

Finley, J. P., et al. 2001, in Proc. 27th Int. Cosmic Ray Conf. (Hamburg), 2827

Fossati, G., Buckley, J., Edelson, R. A., Horns, D., \& Jordan, M. 2004, NewA Rev., 48, 419

Fossati, G., Celotti, A., Ghisellini, G., Maraschi, L., \& Comastri, A. 1998, MNRAS, 299, 433

Fossati, G., et al. 2000a, ApJ, 541, 153 $2000 \mathrm{~b}, \mathrm{ApJ}, 541,166$

Ghisellini, G., Celotti, A., Fossati, G., Maraschi, L., \& Comastri, A. 1998, MNRAS, 301, 451

Ghisellini, G., Tavecchio, F., \& Chiaberge, M. 2005, A\&A, 432, 401

Giebels, B., Dubus, G., \& Khélifi, B. 2007, A\&A, 462, 29

Hickson, P., Auman, J. R., Ninkov, Z., Fahlman, G. G., Walker, G. A. H., \& Menon, T. K. 1982, ApJ, 258, 53

Hillas, A. M., et al. 1998, ApJ, 503, 744

Jahoda, K., Swank, J. H., Giles, A. B., Stark, M. J., Strohmayer, T., Zhang, W., \& Morgan, E. H. 1996, Proc. SPIE, 2808, 59

Jordan, M., et al. 2001, in Proc. 27th Int. Cosmic Ray Conf. (Hamburg), 2691

Katarzyński, K., Ghisellini, G., Tavecchio, F., Maraschi, L., Fossati, G., \& Mastichiadis, A. 2005, A\&A, 433, 479

Krawczynski, H., Coppi, P. S., \& Aharonian, F. 2002, MNRAS, 336, 721

Krawczynski, H., et al. 2001, ApJ, 559, 187 . 2004, ApJ, 601, 151

Krennrich, F., et al. 2001, ApJ, 560, L45 2002, ApJ, 575, L9
. 2003, in Proc. 28th Int. Cosmic Ray Conf. (Trukuba), 2603

\section{REFERENCES}

Lessard, R. W., Buckley, J. H., Connaughton, V., \& Le Bohec, S. 2001, Astropart. Phys., 15, 1

Li, T.-P., \& Ma, Y.-Q. 1983, ApJ, 272, 317

Lockman, F. J., \& Savage, B. D. 1995, ApJS, 97, 1

Macomb, D. J., et al. 1995, ApJ, 449, L99

Maraschi, L., Ghisellini, G., \& Celotti, A. 1992, ApJ, 397, L5

Maraschi, L., et al. 1999, ApJ, 526, L81

Mastichiadis, A., \& Kirk, J. G. 1997, A\&A, 320, 19

Mohanty, G., et al. 1998, Astropart. Phys., 9, 15

Mücke, A., Protheroe, R. J., Engel, R., Rachen, J. P., \& Stanev, T. 2003, Astropart. Phys., 18, 593

Nilsson, K., Pursimo, T., Takalo, L. O., Sillanpää, A., Pietilä, H., \& Heidt, J. 1999, PASP, 111, 1223

Peterson, B. M., Wanders, I., Horne, K., Collier, S., Alexander, T., Kaspi, S., \& Maoz, D. 1998, PASP, 110, 660

Petry, D., et al. 2000, ApJ, 536, 742

Piron, F., et al. 2001, A\&A, 374, 895

Punch, M., et al. 1992, Nature, 358, 477

Rebillot, P. F., et al. 2006, ApJ, 641, 740

Rothschild, R. E., et al. 1998, ApJ, 496, 538

Sambruna, R. M., Maraschi, L., \& Urry, C. M. 1996, ApJ, 463, 444

Sikora, M., Begelman, M. C., \& Rees, M. J. 1994, ApJ, 421, 153

Stecker, F. W., \& De Jager, O. C. 1998, A\&A, 334, L85

Tanihata, C., Urry, C. M., Takahashi, T., Kataoka, J., Wagner, S. J., Madejski, G. M., Tashiro, M., \& Kouda, M. 2001, ApJ, 563, 569

Tavecchio, F., Maraschi, L., \& Ghisellini, G. 1998, ApJ, 509, 608

Tavecchio, F., et al. 2001, ApJ, 554, 725

Ulrich, M.-H., Maraschi, L., \& Urry, C. M. 1997, ARA\&A, 35, 445

Urry, C. M., \& Padovani, P. 1995, PASP, 107, 803

Villata, M., Raiteri, C. M., Lanteri, L., Sobrito, G., \& Cavallone, M. 1998, A\&AS, 130, 305

Von Montigny, C., et al. 1995, ApJ, 440, 525

Zweerink, J. A., et al. 1997, ApJ, 490, L141 